Cahiers de recherches médiévales

\title{
Une édition singulière des œuvres de César
}

L'édition de Godefroy Jungerman (1606)

\section{Monique Bouquet}

\section{(2) OpenEdition \\ Journals}

Édition électronique

URL : https://journals.openedition.org/crm/2569

DOI : $10.4000 / \mathrm{crm} .2569$

ISSN : $1955-2424$

Éditeur

Honoré Champion

Édition imprimée

Date de publication : 30 juin 2007

Pagination : 129-157

ISSN : 1272-9752

Référence électronique

Monique Bouquet, "Une édition singulière des œuvres de César », Cahiers de recherches médiévales [En ligne], 14 spécial | 2007, mis en ligne le 30 juin 2010, consulté le 15 décembre 2022. URL : http:// journals.openedition.org/crm/2569; DOI : https://doi.org/10.4000/crm.2569 


\title{
rin
}

\section{Une édition singulière des ouvres de César : l'édition de Godefroy Jungerman (1606)}

En 1606, Godefroy Jungerman éditait les œuvres de César à Francfort sur Main dans un ouvrage in-quarto, dont Jaucourt, dans une rubrique de l'Encyclopédie ${ }^{1}$, signale qu'il suscita la curiosité.

\author{
C. IVLII \\ CAESARIS \\ QUAE EXSTANT \\ ex \\ nupera Viri docti accuratissima recognitione \\ Accedit nunc \\ Vetus Interpres Græcus librorum VII de Bello gallico \\ ex bibliotheca P. PETAVII \\ Prceterea \\ Notæ, Adnotationes, Commentarii partim veteres, partim noui \\ in quibus Notæ tum Politicæ, tum Criticæ \\ Io. BRANTII Antuerpiani \\ Adhoec \\ INDICES Rerum \& Locorum vtiles \\ Quce altera pagina plenius exhibet \\ EDITIO adornata \\ Opera et studio GOTHOFREDI IVNGERMANI Lipsiensis \\ FRANCOFVRTI \\ Apud Claudium Marnium \& heredes Ioannis Aubrii \\ MDCVI \\ Cum Priuilegio Sac. Caes. Maiestatis, et Regis Gallice \\ L'observation attentive de cette édition (composée de deux volumes \\ conjoints) permet de faire le point sur le statut réservé aux textes de César en ce \\ début du XVII ${ }^{\mathrm{e}}$ siècle, puisque Jungerman produit une nouvelle édition des Com- \\ mentarii tout en leur adjoignant les interprétations et gloses auxquelles ces écrits ont \\ donné lieu au siècle précédent. Malgré une disposition à première vue disparate, \\ l'ensemble est organisé et cohérent. Nous commencerons par décrire globalement \\ l'ouvrage et par présenter son auteur avant de préciser les circonstances de la fabri- \\ cation de ce livre gros de plus de mille pages. Nous nous attacherons ensuite à ob- \\ server les commentaires réunis par l'érudit et à tenter de voir quelle sélection et, en \\ même temps, quels regroupements d'auteurs il a opérés pour produire sa compila-
}

${ }^{1}$ Encyclopédie ou Dictionnaire raisonné des sciences, des arts et des métiers, t. IX, p. 380.

Cahiers de Recherches Médiévales, 14spé, 2007 
tion et apporter un éclairage tant sur la réception de César au $\mathrm{XVI}^{\mathrm{e}}$ siècle que sur celle qu'il paraît privilégier au début du XVII ${ }^{\mathrm{e}}$.

\section{L'ouvrage et son auteur}

L'ouvrage est constitué de deux parties² dont la première (469 pages) contient le texte latin des Commentaires ${ }^{3}$. Ils sont précédés d'une table des matières (portant sur l'ensemble du livre et inscrite au dos de la page de titre), d'une préface adressée à Paul Petau, d'un «Avis au lecteur », de résumés ou summaria - empruntés à Juste Lipse - et de trois cartes géographiques ${ }^{4}$. A la suite des Commentarii, Jungerman reproduit des fragmenta de César et un index rerum et nominum. Ce qui fait l'originalité de cette première partie du volume, c'est que le texte latin des sept livres du De Bello gallico, propres à César, est assorti d'une traduction en grec ${ }^{5}$, l'ensemble étant présenté sur deux colonnes.

La seconde partie (579 pages), outre des index géographiques situés à la fin de l'ouvrage, regroupe des commentaires que Jungerman, à l'exception de notes qu'il a lui-même produites sur la traduction grecque, a empruntés à d'autres éditeurs et qui se rapportent aux textes de la première partie, sans que les expressions glosées soient toujours identiques à celles de ces textes. Une nouvelle page de titre présente sommairement la liste de ces éditeurs ${ }^{6}$, et Jungerman, après un nouvel «Avis au lecteur ", la répète sous la forme d'un index qui fournit quelques précisions sur les auteurs sommairement annoncés? ${ }^{7}$. Les emprunts qu'il opère, constitués essentiellement de notes, annotations, ou commentaires, sont ensuite reproduits selon un ordre que l'éditeur a justifié dans son «Avis au lecteur» et selon des proportions qui varient de l'un à l'autre: certaines notes sont partiellement publiées ${ }^{8}$, d'autres sont précédées d'une biographie d'auteur', d'autres le sont de la Préface de leur auteur,

${ }^{2}$ Que nous signalerons dans nos notes en utilisant les sigles I et II.

${ }^{3}$ La Guerre des Gaules (César-Hirtius), La Guerre civile, La Guerre Alexandrine, La Guerre d'Afrique et La Guerre d'Espagne.

${ }^{4}$ Romani Imperii Typus, Gallice Veteris Descriptio, Typus Hispanice Veteris.

${ }^{5}$ Tous les autres récits de guerre, ainsi que les fragments, sont publiés uniquement dans leur forme latine.

${ }^{6}$ Rhellicanus, Glareanus, Glandorpius, Camerarius, Brutus, Manutius, Sambucus, Vrsinus, Ciacconius, Hotmanus, Brantius. Est ajouté à cette liste un Nomenclator Geographicus duplex, e quibus alter R. Marliani.

${ }^{7}$ Ioan. Rhellicanus Tigurinus - Henricus Glareanus Poeta Laureatus - Ioan. Glandorpius Monasteriensis - Ioach. Camerarius Pabepergensis - Ioan. Mich. Brutus-Aldus Manutius Paulli F. Aldi N. - Ioan. Sambucus - F. Ursinus - P. Ciacconius Toletanus - Fr. Hotmanus I.C.- Ioan. Iucundus Veronensis - Iul. Coesar Scaligerus - Hier. Magius - Adr. Turnebius Ioan. Brantius - Gothofredus Iungermanus Lipsiensis - Raym. Marlianus - Nomenclator Geographicus. L'éditeur précise la part que chacun apporte à son ouvrage, certains ne lui fournissant que leurs explications techniques (machines, ponts), toponymiques ou géographiques.

${ }^{8}$ Du commentaire établi par Camerarius, ne figurent que les notes des livres II et IV de la Guerre des Gaules (II, p.182-84) et celles du livre III de La Guerre civile (p. 184-86), après un Avis qui justifie cette publication réduite (p. 181).

${ }^{9}$ Telle la Vie de Chacon (Ciacconius) que Jungerman a empruntée à A. Schott (II, p. 262-65). 
totale $^{10}$ ou partielle ${ }^{11}$. Enfin, après ses propres annotations sur la traduction en grec des sept livres de la Guerre des Gaules ${ }^{12}$, Jungerman fournit deux ensembles de remarques géographiques empruntées respectivement à Marlianus ${ }^{13}$ et à un Nomenclator Geographicus ${ }^{14}$, leur ajoutant un très bref extrait de Manuzio sur la division de la Gaule ${ }^{15}$.

Si cette présentation, sommaire et technique, laisse apparaître la diversité des composantes de cette seconde partie, elle suggère cependant une organisation dont la cohérence tient à l'intention de Jungerman de fournir au lecteur le plus de données possible, afin d'éclairer sa lecture de César et de lui permettre d'apprécier non seulement la diversité des interprétations des Commentarii jusqu'alors diffusées, mais encore celle de la transmission et de l'établissement du texte latin. Le maître d'œuvre, en effet, a le souci de transmettre un livre dont l'érudition ne le cède pas à une destination utile.

Un maître d'œuvre que signale l'article «Leipsic» de l'Encyclopédie, déjà cité, dès lors qu'il est question des gens de lettres qui ont fait la célébrité de la ville allemande, parmi lesquels les deux frères Jungerman, dont l'un, répondant au prénom de Godefroy, «publia le premier les commentaires de Jules César en grec». L'encyclopédiste ajoute que «cette édition faite à Francfort en in- $4^{e}$ est extrêmement recherchée des curieux». Et c'est une même singularité reconnue à l'ouvrage qu'avait déjà notée la brève rubrique consacrée au littérateur allemand dans le Dictionnaire de Pierre Bayle ${ }^{16}$, informant de surcroît que la publication de la traduction en grec était accompagnée non seulement des remarques de Jungerman lui-même sur le traducteur grec mais aussi de celles de «plusieurs doctes critiques sur les commentaires de J. César». Ce dictionnaire précisait en outre que notre auteur, fils d'un professeur de droit de Leipzig, était, du côté maternel, le petit-fils de Joachim Camerarius de Bamberg ${ }^{17}$. Il avait déjà publié, avant de s'intéresser aux Commentaires de César, une version latine des Pastorales de Longus et était également l'auteur

\footnotetext{
${ }^{10}$ C'est le cas pour Johann Müller (Rhellicanus) qui introduit son commentaire (II, p. 1-4), en attestant qu'il marche sur les pas du grammaticus Servius, lequel recommande que soient précisés au préalable, la vie de l'auteur, le titre de l'ouvrage, son genre, l'intention de l'auteur, le nombre de livres. Recommandation à laquelle se plie Müller.

${ }^{11}$ C'est le cas pour Glaréan (II, p. 98) ou encore pour Glandorp (II, p. 130).

${ }^{12}$ II, p. 537-52.

${ }^{13}$ II, p. $553-71$.

${ }^{14}$ II, p. $572-78$.

${ }^{15}$ II, p. $578-79$.

${ }^{16}$ Pierre Bayle, Dictionnaire historique et critique, Vol. 2 : p. 882-83.

${ }^{17}$ J. Camerarius (1500-1574) est présenté par Du Petit Thouars (Bibliographie universelle ancienne et moderne, Paris, Michaud frères, t.VI, p. 599-602) comme un enfant précoce qui, passionné par le grec, copia, avant l'âge de 18 ans, de nombreux auteurs grecs et, parmi eux, Homère. Le nom de ce «savant universel» dont il serait trop long de citer la liste des ouvrages (et plus particulièrement de ses traductions et commentaires des textes anciens) est à associer à celui de Melanchton, un ami intime, dont il publia une Vie en latin, et avec qui il rédigea la confession d'Augsbourg.
} 
d'une édition annotée du De Equuleo ${ }^{18}$ et d'un ensemble de lettres imprimées. Cette rubrique biographique s'achève sur une remarque particulièrement intéressante pour notre étude : Jungerman était "Correcteur d'Imprimerie dans la maison Wechel ${ }^{19}$. Or, c'est précisément dans cette imprimerie que l'érudit édite sa compilation. Certes le nom de Wechel n'apparaît pas sur la page de titre de cette édition, mais ceux de Claude Marnius et Jean Aubry, qui y figurent, désignent les successeurs et héritiers d'André Wechel $^{20}$. En 1606, J. Aubry étant mort, ses héritiers, associés à Claude Marnius, dirigeaient la maison, anciennement Wechel, pareille à « une sorte de service postal $»$ pour humanistes ${ }^{21}$.

De toutes ces informations nous pouvons déduire que notre érudit, correcteur de l'imprimerie Wechel, dans ce «carrefour humaniste», bénéficiait de contacts nombreux avec tous gens de lettres et peut-être plus particulièrement avec ceux de la Réforme, et que son office lui rendait plus aisée la collecte des éditions de César jusqu'alors publiées.

\section{La fabrique du livre}

La dédicace à Paul Petau ainsi que le premier «Avis au lecteur $»^{22}$ sont riches de renseignements qui expliquent comment Jungerman a pu réaliser son assemblage.

Si la Préface est dédiée à Paul Petau, c'est tout simplement parce que c'est de la bibliothèque de ce dernier que parvient la traduction en grec du De Bello gallico. L'érudit allemand n'ignore pas la notoriété de ce Conseiller parlementaire, célèbre pour sa richesse en livres rares ${ }^{23}$, pour le haut rang qu'il occupe dans la Respublica

${ }^{18}$ On doit le De Equuleo (Le Chevalet) à Girolano Maggi, dit Magius (1523-1572), correcteur d'imprimerie chez Ziletti à Venise. L'édition de Jungerman est de 1608 à Francfort, chez Cl.Marnius \& heredes Aubrii.

${ }^{19}$ Selon l'article que Weiss et Ledru (Bibliographie universelle ancienne et moderne, t. XXII, 1812, p. 152-153) consacrent à ce savant philologue (mort en 1610), Jungerman n'accepta cette fonction que «pressé par les circonstances » ayant jusque-là refusé, en dépit de ses connaissances, une charge dans le barreau ou dans l'éducation. C'est dans cette imprimerie Wechel qu'il se fit connaître pour «de bonnes éditions d'auteurs classiques». L'article souligne la dignité de cette charge de Correcteur, citant Érasme lui-même comme exemple d'érudit qui aimait à «seconder les travaux de typographes classiques». Il signale également la primauté de publication de la traduction grecque en 1606, ajoutant que sa réimpression en 1669 fut moins correcte.

${ }^{20}$ A. Wechel, (1510-1581), fils et héritier de l'imprimeur parisien Chrétien Wechel, fuyant les massacres de la Saint-Barthélémy avait installé son imprimerie à Francfort.

${ }^{21}$ «A kind of humanist postal service». selon R. J. W. Evans : «The Wechel presses: humanism and Calvinism in Central Europe, 1572-1627 ». The Past and Present Society, Oxford, 1975, p. 34.

${ }^{22}$ I, p. $2-4$; I, p. 4-6.

${ }^{23}$ Cet antiquaire (1568-1614) né à Orléans, obtint dès l'âge de vingt ans la charge de Conseiller du Parlement de Paris. Collectionneur d'antiquités et de médailles, il avait aussi une bibliothèque riche d'ouvrages et de manuscrits précieux (provenant pour la plupart de la bibliothèque de Pierre Daniel, après la mort de ce dernier en 1604), et n'hésitait pas à les communiquer. On a gardé de lui, entre autres, un in-quarto, publié en 1610 à Paris qui, outre son portrait, représente vingt-sept planches des principales antiquités de son cabinet: 
litteraria et pour la soif qu'il suscite chez tout un chacun d'accéder à sa fréquentation. Regrettant la distance géographique qui lui interdit cette fréquentation - et prive l'éloge d'être prononcé de vive voix - Jungerman requiert alors de ce dédicataire d'avaliser et d'encourager son projet d'édition. Un éloge quelque peu conventionnel qui, en réalité, en contient un second, plus direct et plus instructif de la fabrique du livre : c'est Bongars qui a fourni à Jungerman l'occasion exceptionnelle d'éditer pour la première fois le texte de la Guerre des Gaules avec, en regard, une traduction grecque ${ }^{24}$. C'est à lui que revient la louange d'avoir remis entre les mains du correcteur de Francfort cette traduction que possédait Paul Petau et de lui avoir suggéré une entreprise audacieuse. Et c'est aussi à Claude Marnius ${ }^{25}$ que revient le projet de confier à Jungerman - avant même l'arrivée de Bongars - le soin de réaliser une édition de César ${ }^{26}$ sous la forme d'un ouvrage copieux et raffiné. Concours de circonstances fortuit et heureux qui a fait se rencontrer à Francfort un légat Français et le correcteur de l'imprimerie Wechel, dirigée par Claude Marnius et alii. Voilà comment notre éditeur a été chargé d'une double mission dont il mesure bien les difficultés et les obstacles. Et il lui faut la solide amitié de Grüter ${ }^{27}$ pour ne pas renoncer à l'entreprise.

Tous ces garants et guides - loués et honorés de tous - ont jugé utile la tâche de Jungerman et lui ont suggéré de joindre à l'édition des textes de César (dont les

\footnotetext{
Antiquarice supellectilis portiuncula. D'après Weiss, Bibliographie universelle ancienne et moderne, t. XXXIII, p. 458). Cf. également le Dictionnaire de P. Bayle, vol. 3 : p. 690-92.

${ }^{24}$ Ibid., (t. V, p. 102-04) : L'article de Tabaraud présente Jacques Bongars (1554-1612) comme le cousin de Paul Petau et comme un calviniste au service du roi Henri IV. Formé aux belles lettres par un anabaptiste, il apprit le droit auprès de Cujas. Pendant près de trente ans, il servit d'ambassadeur au roi Henri IV auprès des cours d'Allemagne, acquérant la réputation d'un savant et d'un honnête homme. Avec Paul Pétau, il se partagea les manuscrits que Pierre Daniel avait récupérés du pillage, par les calvinistes, de l'abbaye de Saint Benoît sur Loire, enrichissant ainsi sa bibliothèque qui contenait bien d'autres ouvrages dont ceux hérités du fonds Cujas. On sait, par sa correspondance, qu'il était ami de Joachim Camerarius (supra, n.17). Cf. également le Dictionnaire de P. Bayle, vol. 1 : p. 604-07.

${ }^{25}$ Ce gendre d'André Wechel était, selon Jungerman, un adepte infatigable de César, ayant également le souci de sans cesse augmenter le fonds de livres rares et d'exception.

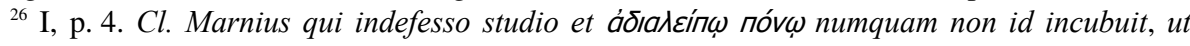
Remp. litterariam rara et eximia supellectile augeat, atque exornet, nuper inter alia animum quoque ad CÆSAREM adiecit.

${ }^{27}$ On retiendra de Grüter (1560-1627) qu'il quitta sa ville natale d'Anvers pour cause de religion. Cet humaniste protestant, après un séjour forcé en Angleterre, revint à Leyde et enseigna dans plusieurs universités allemandes. Il fut longtemps en charge de la Bibliothèque Palatine et de ses manuscrits. Auteur de poésies latines, il produisit de très nombreux commentaires d'auteurs latins et des compilations dont l'une intitulée Lampas siue fax artium liberalium dans laquelle il réunit, selon Boissonade (Bibliographie universelle ancienne et moderne t. XVIII, p. 567-69) «de grands commentateurs et critiques devenus rares de son temps ». C'est au compilateur qu'il était que Jungerman semble faire un clin d'œil dans son Avis au lecteur lorsqu'il déclare suivre l'exemple de cet eximius uir à qui il attribue un rôle d'oracle, ou encore d'éclaireur. Cf. également le Dictionnaire de P. Bayle, vol. 2 : p. 620-23.
} 
sept livres assortis de la traduction en grec) toutes les commentaires produits jusqu'alors.

C'est après avoir informé son lecteur de ces circonstances que Jungerman l'avise de chaque caractéristique de son édition :

Le texte latin des Commentaires dans leur ensemble, est celui que l'officine de François Rapheleng ${ }^{28}$ a publié cette même année 1606, mais dont on ignore le réviseur-correcteur ${ }^{29}$ : en effet, sur la page titre, le nom de celui-ci est tu, tout comme l'était celui de l'édition de 1593, chez le même Rapheleng, et dont on savait cependant qu'il s'agissait de Juste Lipse, trop modeste pour faire imprimer son nom. Jungerman voudrait bien attribuer cette nouvelle édition à Lipse mais il lui semble bien qu'une autre main a procédé à cette nouvelle recension. Quoi qu'il en soit, si la modestie de Lipse et la magnanimité du second réviseur sont louables, il y a surtout et heureusement une lettre du typographe qui invoque la tradition de ses aïeux pour attester que l'établissement scientifique du texte, qui est du dernier poli, est irréprochable. Quant aux fragments qui suivent, ils ne sont pas de la même main, mais ont été établis par un homme érudit et ne souffrent aucune disharmonie avec le texte des Commentaires.

Le traducteur grec pose également problème et si l'éditeur s'interroge sur son nom (Planude ${ }^{30}$ ? un de ses contemporains ? Théodore de Gaza ${ }^{31}$ ) il laisse entrevoir que ce qui compte le plus pour lui c'est l'innovation de cette édition - latin-grecqui procurera au lecteur un intérêt plus vif à lire César. Toutefois il ne manque pas de citer les avis d'éminents hellénistes qu'il a consultés à ce sujet. Scaliger ${ }^{32}$, qui reconnaît qu'il peut se tromper d'après une lettre que cite partiellement Jungerman, considère que le texte ne remonte pas au-delà de Planude. Hoeschel ${ }^{33}$, sans avoir vu le codex grec, croit qu'il est de Planude par simple comparaison avec d'autres textes

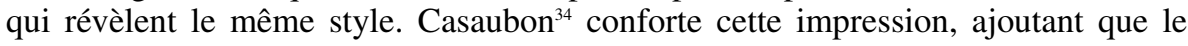

${ }^{28}$ Ce savant orientaliste (1539-1597) enseigna le grec, l'hébreu et l'arabe à l'université de Leyde. Il était le gendre de l'imprimeur Plantin qu'il remplaça à Anvers avant de diriger, à partir de 1585, une imprimerie à Leyde.

${ }^{29}$ Ce pourrait être l'œuvre de Joseph-Juste Scaliger (1540-1609), qui succéda à Juste Lipse à l'université de Leyde: C. Julii Coesaris quce exstant: Ex nova \& accuratissima viri docti recognitione, In Officina Plantiniana Raphelengii, M. D. CVI.

${ }^{30}$ Moine byzantin du XIII ${ }^{\mathrm{e}}$ siècle, qui a traduit en grec des auteurs latins, tels Boèce ou Ovide, et peut-être César.

${ }^{31}$ Philosophe et grammairien byzantin du $\mathrm{XV}^{\mathrm{e}}$ siècle, auteur d'une grammaire grecque : il a traduit, en latin, de nombreux textes militaires grecs et, en grec, quelques textes latins dont le De Senectute de Cicéron le De Bello gallico de César, ou encore le De Re militari de Végèce, auquel se réfèrent souvent bon nombre de commentateurs de César.

${ }^{32}$ Il s'agit de Joseph-Juste Scaliger (1540-1609). Ce philologue protestant a commenté de nombreux auteurs grecs et latins, dont César, et a traduit des épigrammes de Martial en grec. Professeur d'histoire ancienne, il est resté célèbre comme chronologiste.

${ }^{33}$ Humaniste allemand (1556-1617), très féru de grec, éditeur de nombreux auteurs grecs, qui a établi un catalogue de manuscrits grecs.

${ }^{34}$ Ce protestant genevois (1559-1614) a enseigné le grec à Genève avant d'être mandé par Henri IV à Paris pour devenir son bibliothécaire. Il a édité et commenté de nombreux auteurs grecs. 
style simple du traducteur répond bien à la simplicitas de César. Enfin, un ami de Jungerman, Thomas Segheth $^{35}$, en a lu quelques pages et pense aussi qu'il s'agit de Planude. Ce long développement sur le traducteur grec, outre qu'il réunit d'autres humanistes de l'entourage de Jungerman que ceux cités dans la Préface et au début de l'Avis, atteste le souci d'érudition de ce dernier qui, fort de son innovation, annonce qu'il va proposer en fin d'ouvrage ses propres annotations de la traduction grecque :

- la numérotation est celle de l'usage moderne.

- le sommaire des livres des Commentaires a été emprunté à Juste Lipse.

- les fragments qui suivent la Guerre d'Espagne, sans nom d'auteur, ont pour origine Plutarque : c'est ce que signale le codex de P. Petau, alors que celui de Cujas n'en dit rien.

- les cartes proviennent d'ateliers lyonnais.

Et Jungerman d'annoncer des manques - dont les notes hypercritiques de Grüter qui viendront un peu plus tard et à part - ainsi que quelques erreurs dues à la hâte : sur ce point, il se compare à César lui-même «qui devait tout faire en même temps chez les Nerviens $»^{36}$.

De toute évidence l'éditeur-correcteur d'imprimerie a le souci de proposer un texte latin irréprochable et de démontrer sa maîtrise du grec, qu'il partage avec de savants hellénistes. Il n'oublie pas, dans le même temps de signaler les rapports qu'il entretient avec ces savants, qu'il mentionne au gré du sujet. Cet «Avis au lecteur», depuis le commanditaire et imprimeur Claude Marnius, qui ne ménage ni ses conseils ni sa dépense, jusqu'à l'ami Segheth à ne pas laisser sur le bord du chemin humaniste, foisonne d'indications qui mettent en interaction les relations humaines, les échanges d'ouvrages précieux et de remarques critiques sur l'établissement scientifique des textes, laissant supposer que la maison Wechel constitue un centre actif de la Respublica litteraria.

\section{Le suntagma des commentaires aux ouvres de César.}

Le second «Avis au lecteur» qui introduit la seconde partie de l'ouvrage vient compléter les informations sur la vie intellectuelle de l'imprimerie Wechel et sur les choix opérés par son correcteur en matière d'éditions commentées de César. Une sélection disparate à première vue et à plusieurs égards : les auteurs qualifient diversement leurs remarques - notce, adnotationes, commentarii - et, lorsqu'ils sont proches du modèle de l'exégèse antique, ils n'hésitent pas à fournir une analyse très subjective de telle ou telle expression relevée dans le texte césarien ; certains intègrent à leurs scolies des figurce, quand celles-ci ne constituent pas une sorte de livret à part; d'autres se cantonnent à la tradition manuscrite et Jungerman peut bien ne leur emprunter que des morceaux d'ouvrages. Bref, ces commentateurs anciens et modernes sont associés par Jungerman de façon originale dans un ensemble dont la

${ }^{35}$ De ce Thomas Segheth, il reste essentiellement un recueil de divers traités De Principatibus Italice. Sa participation à un ouvrage contenant des principes philosophiques et médicaux de l'Antiquité - Venerandce Antiquitatis: Assyriorum, Chaldceorum, Persarum, Aegyptiorum, Arabum \& Grcecorum, ... philosophica et medica principia - laisse supposer qu'il était un érudit connaissant bien le grec.

${ }^{36}$ César, B.G. II, 20 : Caesari omnia uno tempore erant agenda. 
cohérence repose sur une disposition, délibérément chronologique, qui permet de suivre la diffusion des Commentaires au cours du $\mathrm{XVI}^{\mathrm{e}}$ siècle depuis le commentaire à l'antique de Müller pratiqué, dès 1529, jusqu'au mode nouveau d'annotations (politiques et critiques) de Johannes Brandt (Brantius) en 1606. Et c'est ce parcours d'une transmission variée et continue des Commentaires de César qui peut intéresser le lecteur moderne, qu'il soit soucieux de jauger la dimension idéologique des uns et des autres, de découvrir des «apparats » critiques procédant de lectures de manuscrits rassemblés pour l'occasion plutôt que constitués en «familles », ou de considérer le statut même du texte de César du point de vue de sa latinité ou du «prétexte » auquel il peut être réduit.

Suivons donc ce second Avis en étayant les indications de Jungerman de certaines remarques analytiques ${ }^{37}$. C'est Johann Müller (Rhellicanus) qui marche à la tête de cette louable armée de commentateurs ${ }^{38}$; son commentaire n'a été publié à Bâle qu'en 1543, mais il en a enseigné le contenu, dès 1529, à ses étudiants de Berne. Ce pasteur luthérien (1473-1542), également poète, qui enseignait la théologie à Berne, revendique lui-même cette première place, dans une préface datée de 1540 et à l'extrême fin des scolies du livre I de la Guerre des Gaules ${ }^{39}$. Jungerman le produit donc en premier, reconnaissant toutefois une primauté de publication à Henri Glaréan ${ }^{40}$ qui, dans la préface de son César, souscrit la date de 1538. Mais, selon notre éditeur, les commentaires de Glaréan seraient restés en souffrance pendant quelques années. Ne s'attardant pas sur la préséance à accorder à deux gloires du pays Helvète qui, s'ils renaissaient, ne rivaliseraient pas à ce sujet, Jungerman précise que c'est Bongars - louable en tous lieux - qui lui a apporté l'ouvrage de Müller, ouvrage qu'il a amendé tant était fautive cette première édition, le nombre d'erreurs dépassant le nombre de pages! Pour ce qui est de l'ouvrage de Glaréan, Jungerman dit avoir utilisé le codex que possédait Grüter, riche des révisions et des ajouts apportés par l'auteur à la première édition, avant une nouvelle publication,

\footnotetext{
${ }^{37}$ Comme nous l'avons dit plus haut, un index situé après l'Avis au lecteur, fournit quelques précisions sur les sources compilées par l'éditeur. Nous le retenons dans notre présentation tout en l'enrichissant de données extérieures.

${ }^{38}$ II, p. 2 : Agmen itaque hoc laudabile ducere Rhellicanum siui.

${ }^{39}$ Ibid., Ait enim extremis Adnotationibus ad l. i de bel. gallico se "primam hanc foeturam Bernensibus discipulis dictasse anno MDXXIX».

${ }^{40}$ Glaréan (1488-1563), né dans le canton de Glaris - d'où son nom - participa activement à la vie sociale et politique de Fribourg en Brisgau, ville qu'il choisit de rejoindre après avoir quitté Bâle où il enseignait les mathématiques et la philosophie. Cette retraite s'expliquait par son refus de participer aux troubles religieux de la cité helvète. A Fribourg, il enseigna l'histoire et la littérature, mais se distingua aussi par les poèmes latins qu'il composa et qu'il chanta à la cour, en s'accompagnant d'instruments de musique. Féru de musique, il se distingua également par son goût pour la géographie - ancienne et nationale - ce qui lui inspira des ouvrages traitant de cette discipline. A toutes ces activités s'ajoutaient les nombreuses annotations qu'il publia sur les auteurs latins et grecs. Ami d'Érasme, il subit la froideur, voire la jalousie de ce dernier, tant il passait pour un esprit profond en histoire comme en antiquités gréco-romaines (d'après l'article de Weiss dans Bibliographie universelle ancienne et moderne, t. XVII, p. 493-94.).
} 
chez Gravius, en 1544, à Fribourg. Vient ensuite Glandorp de Leipzig ${ }^{41}$ dont le César, publié en $1574^{42}$, par le soin du luthérien Reineccius et de l'imprimeur A. Schneider dans l'officine Vögelin ${ }^{43}$, a été fourni à Jungerman par son propre frère, qui l'avait en sa possession, et dans lequel il a remarqué, comme dans l'ouvrage de Glaréan, de nombreuses erreurs dues aux apprentis typographes; c'est de nouveau une entremise familiale ${ }^{44}$ qui lui a permis de disposer de quelques notes retrouvées dans les écrits de son grand-père maternel, Joachim Camerarius.

La présentation de ces premiers commentateurs nous permet de situer Jungerman lui-même dans son environnement immédiat: le correcteur profite de sa rencontre avec le providentiel Bongars, de l'amitié fidèle de Grüter et de l'aide familiale de son frère, de son cousin, qui semblent bien mettre tout en œuvre pour la réussite de l'entreprise.

À la suite ce regroupement est proposé un petit livret de uarice lectiones que Jungerman a emprunté, sans nom d'auteur, au César publié par Ch. Plantin, typographe royal d'Anvers, en $1574^{45}$, et dont il ne doute pas qu'il soit de Gian Michaele Bruto $^{46}$; en effet, la page de titre de l'édition, quand bien même la préface n'évoque pas le libellus en question, annonce les scolies de Bruto et, parmi les codices cités en tête du libellus, figure le $F$ impressus LX abhinc anno Florentio ${ }^{47}$. Jungerman qui ne

\footnotetext{
${ }^{41}$ Glandorp (1501-1564), disciple de Mélanchton, bien instruit en langues anciennes, se consacra d'abord à la théologie. Il est connu pour son argumentation virulente contre les anabaptistes, en 1533, mais surtout pour le rayonnement qu'il connut auprès de ses étudiants à Goslar, alors qu'il enseignait la littérature et l'histoire. (D'après l'article de Weiss dans Bibliographie universelle ancienne et moderne, t. XVII, p. 489-90).

${ }^{42}$ Ibid., Glandorp a publié en 1551 une Silua carminum elegiacorum in errationem Commentariorum C. Julii Cesaris de Bello gallico et ciuili. Mais ses Notes sur les Commentaires ont été publiées à titre posthume (il est mort à Marburg en 1564) par son fils Ambroise, qui publia aussi l'Onomasticon historice romance de son père.

${ }^{43}$ Là où Camerarius publia la Vie de Melanchton.

${ }^{44} \mathrm{C}^{\prime}$ est son cousin germain maternel, Ludovicus Camerarius, qui les lui a procurées.

${ }^{45}$ Ce nouveau César était augmenté par rapport celui de 1570, chez le même imprimeur, des scholies de Manuzio et des spicilegia de Zsámboki.

${ }^{46}$ G.-M. Bruto (1517-1592) avait, dès 1564, publié un César, corrigé par P. Manuzio, à Venise, dans l'imprimerie aldine. Selon le rédacteur de la notice qui lui est réservée dans le Dictionnaire de P. Bayle (vol. 1: p. 687-89), ce natif de Venise était «un fort bon Humaniste», qui voyagea, souvent contraint, en Espagne, en Angleterre, en France, en Transylvanie, en Pologne (où il mourut). Il était alors à l'affût des manuscrits qu'il pouvait déterrer avant de «les mettre sous presse». Sur sa vie, cf. aussi la notice du Dizionario biografico degli italiani, Istituti della Enciclopedia italiana, Roma, 1996, t. XIV, p. 730-34, ou encore l'article de Roquefort, revu par Ginguené, dans la Bibliographie universelle ancienne et moderne, t. VI, p. 165-67.

${ }^{47}$ En réalité, c'est à Massa en Toscane, alors qu'il revenait d'Espagne à Venise, en 1558, qu'il avait «déniché » ce manuscrit. Jungerman établit un lien avec Florence, sans doute pour la renommée que valut à Bruto son Histoire de Florence.
} 
connaît pas les conditions d'édition du libellus ${ }^{48}$, dit savoir du moins celle du codex, soit 1512.

A la suite du cahier de Bruto, sont reproduits les travaux de deux autres philologues-codicologues, Aldo Manuzio et János Zsámboki (dit Sambucus). Les scholies du premier appartiennent à une édition des Commentaires de César dont la préface, adressée à Jérôme Matheus, a été écrite en 1570. Cet Aldo Manuzio, petitfils d'Aldo Manuzio, fils de Paulo Manuzio et son successeur à l'imprimerie vaticane, s'inscrit dans la droite ligne tracée par ses deux ancêtres dont le souci était de transmettre de façon irréprochable les textes des auteurs classiques, dont Césara ${ }^{49}$. Quant au second, médecin, historiographe de Maximilien II, numismate, célèbre pour ses Emblemata, il manifestait un intérêt tout particulier à la collecte et à la conservation de manuscrits. Jungerman ne dit rien de ces deux auteurs si ce n'est qu'il a emprunté leurs scolies à l'édition de 1574 de Plantin, celle que Claude Marnius lui a fournie et où ces scolies étaient associées aux leçons de Bruto.

$\mathrm{L}^{\prime}$ ' Avis au lecteur» se poursuit avec la mention conjointe de Fulvio Orsini ${ }^{50}$, dont Jungerman signale que les notes sont à l'image de leur auteur, c'est-à-dire eximice, et Pedro Chacon (Ciacconius) $)^{51}$ dont il reproduit au préalable la biographie

\footnotetext{
${ }^{48}$ Bruto retourna à Lyon dès lorsqu'il apprit qu'il figurait sur la liste des hérétiques, dressée par son ancien éditeur, P. Manuzio, devenu imprimeur officiel du Vatican, et, de Lyon, «selon toutes les apparences il alla à Bâle». C'est ce que précise la notice du Dictionnaire de Pierre Bayle (p. 687-89) qui fournit également des indications sur la composition de son César: l'ouvrage de Bruto «ne consistait point uniquement en Scholies et en uarice lectiones». Il en donne une idée plus avantageuse dans le Passage que je vais copier: Habeo in manibus Casaris commentarios, multis a me animaduersionibus emendatos, quibus iustum uolumen accedet, in quo, certo ordine, politissimi scriptoris uoces phrasesque omnes, tum, quod permagni faciendum est, rerum omnium descriptiones in locos communes redactce habentur: ut si cui scribenda historia, et lauta supellex, et luculenta ex tanti scriptoris monumentis, ad ea ornanda atque illustranda quce uelit, suppeditentur. Eum librum Basilce excusum animus est inscribere Transylvanice principi. Cet extrait d'une lettre de Bruto atteste donc qu'il a assorti la publication de son César de scolies, du libellus retenu par Jungerman et vraisemblablement d'un cahier de loci communes, imprimé à Bâle, avec une page de titre faisant figurer le nom d'Étienne Battori, Prince de Transylvanie auprès de qui Bruto s'est rendu en 1574 pour y composer une Histoire de la Hongrie. Assurément, ces loci communes remettent en mémoire l'utilisation des Commentarii, prévue par Cicéron, dans son Brutus, mais ils instruisent surtout sur une édition variée et augmentée en l'espace de dix ans.

${ }^{49}$ Dès 1513, Aldo Manuzio senior édite les Commentaires de la Guerre des Gaules à Venise.

${ }^{50}$ Fulvio Orsini (1529-1600) fit office de bibliothécaire auprès du cardinal Farnèse ; passionné d'antiquités, il fit don du cabinet de médailles, bronzes et tableaux qu'il s'était constitué au neveu de ce cardinal. Il possédait aussi de nombreux manuscrits qu'il laissa à la bibliothèque du Vatican et, en tant que philologue, il laissa des Notes sur divers auteurs classiques, s'intéressant autant aux traités d'agriculture et à l'historiographie qu'aux œuvres de Cicéron. (D'après l'article de Weiss dans Bibliographie universelle ancienne et moderne, t. XXXII, p. 177-78).

${ }^{51}$ Ce prêtre espagnol, natif de Tolède (1527-1581), formé à la théologie et à la philosophie, fut chargé par le pape Grégoire XIII de réviser la Bible, les Pères, le décret de Gratien et de corriger le calendrier gravé sur marbre au temps de Jules César et installé à la bibliothèque
} 
composée par Andreas Schott ${ }^{52}$. Les notes de ces deux auteurs ont auparavant été confondues, dit-il, et de surcroît, avec celles de bien d'autres commentateurs ${ }^{53}$ : Fulvio Orsini l'atteste, lui qui a détaché de ce mélange les fragmenta ueterum historicorum d'Antonius Augustinus ${ }^{54}$ et les notce de Chacon. Or, ce sont ces notce, détachées, qui, de Rome, sont arrivées chez Claude Marnius en 1606 par l'entremise d'A. Schott $^{55}$. Jungerman intègre donc à son ouvrage les deux ensembles de scolies, d'Orsini et de Chacon, mais prétend ne pas avoir l'impudence de marcher sur les terre de Johannes Brandt qui a l'intention de les associer aux siennes ${ }^{56}$.

La disposition chronologique implique une remontée vers le nord avec François Hotman ${ }^{57}$. C'est à une édition genevoise des notes «renouvelées » d'Hotman que se réfère Jungerman ${ }^{58}$. Et c'est encore Grüter qui lui a procuré ces notes, que la malveillance inepte de quelques uns dénonçait. Notre éditeur y a ajouté des Figurce d'Hotman, augmentées de quelques autres dessins de ponts césariens ou d'ouvrages techniques, empruntés à Giocondo, Scaliger, Maggi, Turnèbe, les uns et les autres

Farnèse. La rubrique rédigée par Villenave (Bibliographie universelle ancienne et moderne, t. VII, p. 610-13) ajoute à la suite de ces informations que, selon Antonius Augustinus, «il semblait né pour corriger et rétablir les auteurs anciens ».

${ }^{52}$ II, p. 262-63. Le jésuite Andreas Schott (1552-1629), dans sa biographie de Chacon, prend pour fil conducteur les activités de réviseur du prêtre espagnol, constituant ainsi un index de ses œuvres, et termine cette biographie par un portrait moral élogieux : aimant être seul pour ne s'occuper que de livres, Chacon participait peu aux échanges collectifs. Il ignorait les honneurs et l'ambition, fréquentait peu les philosophes, recherchant plutôt l'amitié de ceux qui le soutenaient dans ses activités, tels Latinus Latinius et Fulvio Orsini.

${ }^{53}$ Antonius Augustinus, Octauius Panthagatus, Gabriele Faerno, Marc Antoine Muret, Latinus Latinius.

${ }^{54}$ Ce juriste espagnol (1516-1586) après être passé de l'université de Saragosse à celle de Bologne, a été ambassadeur des papes Paul III, Jules III et Paul IV. Intéressé par le droit civil et la matière ecclésiastique, il n'a pas manqué d'éditer des textes d'auteurs latins, notamment de Festus et surtout de Varron.

${ }^{55}$ Dans son Avis au lecteur (II, p. 3), Jungerman rend un hommage appuyé à l'esprit infatigable et libre de Schott, l'invitant à toujours conserver ce zèle et cette liberté.

${ }^{56}$ Johannes Brandt, en effet, dans un Avis au lecteur (II, p. 417) dit bien qu'il est le premier à mettre en lumière (primi in lucem damus) les notes de Chacon, qu'il s'est procuré grâce à A. Schott de la Société de Jésus et dont il dit qu'il ne veut pas priver son lecteur d'un tel «trésor». Brandt évoque bien le mélange des notes de l'espagnol avec celles de bien d'autres, dont celles d'Orsini, qu'il laisse délibérément de côté : il considère en effet qu'Orsini a fait siens les commentaires de Müller, ne sachant si Orsini les a reconnus comme emprunts ou s'il s'agit d'un « vol».

${ }^{57}$ Né à Paris en 1524, Hotman, préférant à l'exercice du barreau l'étude du Droit Romain et des Belles-Lettres, «goûta les nouvelles opinions, pour lesquelles on faisait mourir beaucoup de gens dans le Royaume», lit-on dans le Dictionnaire de P. Bayle (vol. 1: p. 607). Ce littérateur et juriste, qu'il fût en France, en Allemagne ou en suisse, manifesta son engagement aux côtés des Huguenots jusqu'à sa mort, à Bâle en 1590.

${ }^{58}$ Toutefois, lorsqu'il reproduit les notce, il emprunte une partie de la Préface à l'édition, plus connue, publiée à Lyon en 1544 ainsi qu'un excerptum de l'Itinéraire d'Antonin. 
étant fort utiles à tout lecteur soucieux de bien comprendre César ${ }^{59}$. Le choix de l'édition genevoise et la volonté de remédier à la calomnie dont a souffert Hotman signifient vraisemblablement le voeu de Jungerman, toujours soutenu par son fidèle Grüter, de soutenir les Réformateurs et de leur rendre justice en cas d'accusations injustes, touchant notamment à leur interprétation des textes.

Enfin, c'est Johannes Brandt (1550-1639) qui ferme l'agmen des commentateurs sollicités par Jungerman, à propos de qui il concède qu'il «n'est pas besoin de parler de ses notes car l'ouvrage parlera de lui-même ${ }^{60} \gg$ sans rien ajouter à cet éloge. De ce juriste Anversois, on sait qu'il quitta sa ville en raison des troubles qui l'agitaient et qu'il voyagea en France, en Italie, avant de revenir à Anvers où il fut nommé Secrétaire de la ville en 1591. Van Erborn, dans une brève notice ${ }^{61}$, retient de lui qu'il était un «savant modeste et plein d'urbanité», qu'il publia une Vie de Philippe Rubens $^{62}$, des Notes sur les Commentaires de César et des Elogia Ciceroniana Romanorum domi militiceque illustrium.

Quant aux notes de Jungerman lui-même ${ }^{63}$, qui portent uniquement sur la traduction de l'interprète grec (donc sur le De Bello gallico), elles sont l'œuvre d'un annotateur qui a le manuscrit en mains, observe tout ce qu'il comporte de ratures, de surcharges, de notes marginales et, à partir de ces observations, juge de la qualité de la traduction, en se référant toujours au texte de César et au style, sans fioritures, qu'on lui connaît ${ }^{64}$. Ces notes semblent constituer un faire-valoir de notre éditeur, satisfait d'exposer sa connaissance érudite du grec, et, du fait de leur rédaction simultanée de la compilation, elles participent d'une «modernité » que les notes de Brandt - comme nous allons le voir plus loin - révèlent également dans leur association au suntagma des emprunts faits au siècle précédent.

\section{Pourquoi et comment sont commentées les æuvres de César?}

La disposition chronologique des éditions commentées ou annotées des œuvres de César n'empêche pas la grande variété des finalités et pratiques : bien plus, elle permet de révéler les mentalités des éditeurs qui tantôt intègrent une leçon de manuscrits à une paraphrase explicative, tantôt proposent une suite de remarques relevant de la seule édition critique, tantôt choisissent de distinguer, dans une certaine complémentarité, les deux approches. Elle donne à voir une série éditoriale qui laisserait à penser que, de 1538 à 1606, à la pratique du commentaire hérité de l'usage antique se serait substitué un intérêt grandissant pour l'établissement critique du texte avant que les deux modalités, à l'aube du XVII ${ }^{\mathrm{e}}$, soient assumées de façon distincte par un même éditeur, Johannes Brandt.

Le mode retenu par chaque éditeur est quelquefois précisé au début de son commentaire mais, dans la mesure où Jungerman ne choisit pas toujours de repro-

\footnotetext{
${ }^{59}$ Le manque de temps, dit-il, ne lui a pas permis d'ajouter les dessins commentés de bien d'autres (dont Alberti, Budé, Buteo...).

${ }^{60}$ II, p. 3. Un ouvrage qui repose sur des choix bien sûr et ignore la négligence : opus nimirum edecimatum et quod impolitiam nescit.

${ }^{61}$ Biographie universelle ancienne et moderne, t. V, p. 504.

${ }^{62}$ Frère du peintre.

${ }^{63}$ II, p. 537-52.

${ }^{64}$ II, p. 537.
} 
duire les propos liminaires de ses sources, il apparaît que certains des commentaires de sa compilation sont livrés de façon brute et que le lecteur fait lui-même l'expérience autant des notes reproduites que du chemin sur lequel le commentateur l'engage dans l'observation du texte latin. C'est selon ce mode privilégié par chaque éditeur que nous avons pu, dans notre propre observation de l'ouvrage, opérer - sans contrarier la disposition chronologique de l'éditeur - un regroupement typologique tout en mesurant la singularité de chacun. D'un côté les commentateurs qui renvoient de près ou de loin à l'enarratio antique réservée au grammaticus, de l'autre ceux qui s'en tiennent à la consultation de manuscrits afin de proposer des lectiones uarice élaborées de façon comparative et/ou dérivative, de l'autre encore ceux qui s'intéressent aux deux approches textuelles ${ }^{65}$.

Le commentaire de Müller, qui, comme nous l'avons dit, occupe la première place $^{66}$, ressortit surtout à l'onomastique qui prend une part importante dans les 116 rubriques consacrées au livre VII. Pour remédier à l'incurie des libraires qui ont produit le De Bello gallico, le commentateur helvétique met en effet un soin scrupuleux à préciser les lieux et les peuples en se recommandant des auteurs antiques, spécialistes de la question (Pline, Strabon, Pomponius Mela) comme de la tabula Ptolemcei, ou encore de Marlianus et de certains contemporains géographes : il souligne la variation qui affecte un même nom dans l'ensemble de l'œuvre césarienne, sa différence avec la donnée de Ptolémée ou celles des autres éditions, mais c'est pour mieux mettre en évidence la dénomination vulgaire (uulgo) ou française ( $g a l$ lice) qui instruit directement le lecteur dans ses déplacements en Gaule celtique. Le souci d'instruire est premier chez ce commentateur «servien» et c'est la raison pour laquelle les rubriques ne se limitent pas à un leitmotiv de collectionneur ou de spécialiste mais s'emploient à éclairer l'ensemble du texte. Comme le grammaticus, il entend lever toute confusion, toute ambiguité, quel que soit le domaine auquel se rattache l'expression commentée : militaire, historique, géographique, institutionnelle, ethnographique. Si la formule césarienne manque d'évidence, il recourt à l'homonymie ou l'explique brièvement à l'aide de la formule id est ${ }^{67}$, s'il la juge elliptique, il supplée, introduisant sa résolution avec l'impératif supple ${ }^{68}$. Si elle est trop technique, il fournit une fiche plus ou moins détaillée, appuyée sur des auteurs anciens de référence ${ }^{69}$, sans pour autant détacher le lecteur de sa propre réalité. En-

\footnotetext{
${ }^{65}$ Notre observation s'est limitée aux notes que chaque commentateur a produites sur le livre VII de la Guerre des Gaules. Notre intention première était de ne considérer les commentaires que sous un angle stylistique et ce livre VII, du fait de sa grande diversité typologique, devait s'y prêter mieux que les autres. Le résultat de cette observation, qui a mis en évidence que l'elegantia de César n'était pas la préoccupation première des annotateurs, justifie notre choix de mettre plutôt en lumière des caractéristiques propres à chacun d'eux, dont nous pensons qu'elles ne trahissent pas les intentions et pratiques exégétiques relatives aux autres livres.

${ }^{66}$ Io. Rhellicanus, In Librum septimum De Bello gallico Annotationes, II, p. 42-8.

${ }^{67}$ II, p. 44. Perpetuis (B.G. VII, 23), id est continuis trabibus.

${ }^{68}$ II, p. 47. Respicite finitimam Galliam (B.G. VII, 77), supple, Narbonensem.

${ }^{69}$ II, p. 44. Pedes quadragenos (B.G. VII, 23), hoc est, ut singulce materice singulis trabibus quadragenos pedes longis reuinctce essent. Aries autem (Vegetio lib. IV, ch. 14 teste) testudinis caput ferro munitum dicitur quod durissimam frontem habeat, qua muros subruit, uel quod more arietum retrocedit, ut cum impetu uehementius feriat. Hac ille. Porro
} 
fin, si elle est imprécise, il apporte tous les compléments géographiques ou historiques nécessaires, les empruntant à des passages de César lui-même ou à sa propre érudition. Et, à l'occasion, il procède à des remarques stylistiques. Assurément la résolution de l'ellipse par la supplétion témoigne de la breuitas de César, la définition technique d'un appareil militaire fournit la preuve de son style pur et dénué de fioritures, vu que son choix du mot exact lui permet d'aller directement à l'essentiel, la réécriture d'une expression atteste sa maîtrise d'un oratorium ornamentum, tel celui de l'occupatio soigneusement prêtée à Vercingétorix qui devance les objections des siens avant de mettre fin à son discours ${ }^{70}$. Alors que le commentaire de Müller peut paraître éclaté et superficiel, il suppose une finesse d'analyse propre à mettre en évidence non seulement le sens du texte mais aussi la qualité de son expression. Si les adverbes mélioratifs sont absents de ces rubriques, les quelques remarques attestent plus une reconnaissance de l'éloquence de César qu'une emendatio de son texte. En effet, le commentateur va au-delà de la simple observation de l'ordo artificialis césarien, s'il en est, pour souligner l'usage, naturel, d'une construction stylisée (construction grecque ${ }^{71}$ ) ou mieux encore l'habileté rhétorique de celui qui, tout jeune, plaida contre Dolabella: ainsi, alors que la situation nécessite un discours de reproche, César recourt habilement à l'obiurgatio que sa connaissance des troupes sait toutefois nuancer ${ }^{72}$ et lorsqu'un chef gaulois, Critognat, se doit de persuader ses soldats, le discours que lui prête César atteste une suasoire en bonne et due forme : sans négliger le mos maiorum, il procède à une amplification, en allant du plus petit au plus grand et en comparant les prises de position ${ }^{73}$.

Ammianus Marcellinus lib. 23 eum hoc modo describit. « Hinc ad arietem ueniemus. Eligitur abies uel ornus excelsa, cuius summitas duro ferro concluditur et prolixo, arietis efficiens prominulam speciem, quce forma huic machinamento uocabulum indidit, et sic suspensa utrimque transuersis asseribus et ferratis quasi ex lance uinculis trabis alterius continetur, eamque quantum mensurce ratio patitur multitudo retro repellens rursus ad obuia quceque rumpenda protrudit ictibus ualidissimis instar adsurgentis et cedentis arietis qua crebritate uelut reciproci fulminis impetu cedificiis scissis in rimas concidunt structurce laxatce murorum. Hoc genere operis si fuerit exserto uigore discussum, nudatis defensoribus ideoque solutis obsidiis ciuitates munitissimce recluduntur». Hactenus Ammianus. Porro quantum ex utriusque authoris descriptione intelligimus, machina illa bellica non dissimilis ei fuit quce apud maiores nostros ante bombardas inuentas, a felis similitudine Cattum uocabatur. Vtroque enim instrumento moenia concuti solebant, ac dirui. Quo autem alio contrario instrumento aries repelli potuerit, idem Ammianus post citata modo uerba indicat, qui uolet, eum legat.

${ }^{70}$ II, p. 44. Haec si grauia, aut acerba uideantur etc. (B.G. VII, 14). Occupatio est, quasi dicat : scio uobis grauia uideri qua consulo : at interim cogitate multo grauiora, adde etiam inhonestiora esse, qua uobis patienda erunt, si meum consilium neglexeritis.

${ }^{71}$ II, p. 44. Perstare in sententiam uiderunt (B.G. VII, 26). Rursus Grceca dicendi forma usus est, pro in sententia.

${ }^{72}$ II, p. 45. Postero die Caesar contione aduocata (B.G. VII, 52). Oratio est obiurgatoria, in qua tamen obiurgationis acerbitatem temperat laudis admixtione, ut eos ab ipso ne tantillum quidem alienet.

${ }_{73}^{7 I}$ II, p. 47. Nihil inquit de eorum sententia (B.G. VII, 77). Oratio ista Critognati generis est suasorii. In qua primum aduersariorum sententias ualidis rationibus diluit. Deinde cum 
Voilà un commentaire, proche du mode antique, qui propose au lecteur contemporain des explications fournies, notamment en matière d'onomastique ou de toponymie. Le texte de César est actualisé en sa qualité de référent - d'autant que les cartes et indices géographiques que l'ouvrage de Jungerman fournit en son début et en sa fin permettent de suivre concrètement le parcours des armées gauloise et romaine. Müller ne s'interdit pas, dans le même ordre de pensée, de commenter l'orthographe des mots latins en citant d'autres éditions (celle de Manuzio, de Gryphius ou de Beroaldo ${ }^{74}$ ) tout comme il cite, dans ses explications, d'autres savants humanistes, tel Guillaume Budé. Ainsi donc, le besoin de commenter une lectio retenue par un autre n'est pas totalement absent de ce commentaire; toutefois l'intention première est bien de fournir au lecteur le plus d'éclaircissements possibles sur tous les passages ou termes obscurs qui ressortissent au sens du récit césarien.

Les annotations de Glaréan attestent un même intérêt pour l'onomastique. Jean-Claude Margolin a démontré le soin tout particulier que le commentateur prêtait à cette discipline, suggérant que ce soin se justifiait par une volonté tenace de rappeler l'importance de sa patrie d'origine, Glaris, ce «patriotisme helvétique, linguistico-géographique » étant manifeste dans le commentaire du livre I ${ }^{75}$. Il l'est tout autant dans celui du livre VII ${ }^{76}$, même s'il n'y a que 30 rubriques, assez longues il est vrai. Glaréan dénonce l'incurie de César lui-même qui note différemment les noms de peuples, mais surtout celle des copistes ou éditeurs qui, dans leurs codices, les présentent coniunctim ou distinctim, ou encore modifient une lettre, au point que d'un seul peuple on aboutit à deux (Lemouices / Leuouices). Pour lui, l'attention à l'onomastique est indispensable : ainsi en va-t-il de Gebenna par exemple qui désigne un mons et non pas la ville de Genève (Geneua). Mais au-delà de son leitmotiv patriotique, on perçoit l'intérêt qu'il porte à l'exactitude du texte de César: il s'interroge sur l'absence d'une préposition (en regard de l'usage de son époque) et constate qu'elle est restaurée dans certaines éditions ; il tente d'expliquer un mot, tel celui qui désigne la disposition en quinconce ${ }^{77}$, et développe son explication s'il le

subiungit, nempe sibi consultum uideri, ut omnia citius, et patiantur, et designent, quam aut se hostibus dedant, aut per eruptionem et se, et totam Galliam in discrimen coniiciant. Tametsi enim suum consilium de deuorandis hominibus, qui bello inutiles sunt, prima fronte satis crudele sit: tamen id toti Gallice et honestum, et frugiferum fore, si assentiantur. Ne autem tam immane consilium exemplo carere putent, id quoque a maioribus suis captum esse probat: quod per locum a minore ad maius, et per collationem partium inter sese amplificat. Déjà dans une note précédente, Müller avait analysé l'exhortation de Litivaccus (B. G. VII, 38 ) du point de vue des règles de la suasoire.

${ }^{74}$ II, p. 47. Ab infimo solo (B.G. VII, 73) : Exemplar Aldinum et Wolsianum hic perquam corruptum est, post uocem enim pedes omittit, treis et pro occultabantur (ut Beroaldinus et Gryphianus codex habet) exculcabantur legit.

${ }^{75}$ J.-Cl. Margolin, "Glaréan, commentateur du De Bello gallico», dans Présence de César, R. Chevallier éd., Belles Lettres, Paris, 1985, p. 212.

${ }^{76}$ H. Glareanus, In Librum VII De Bello gallico Annotationes. II, p. 118-23.

${ }^{77}$ II, p. 121. Ante hos obliquis ordinibus in quincuncem dispositis (B.G. VII, 73). Glaréan s'intéresse au terme quincuncem qu'utilise César, pour désigner un quincuncialis ordo dont la prononciation difficile explique que l'usage lui ait préféré le terme quincunx. 
considère comme exact, chargé de sens et appartenant à l'usus frequens. Il va même jusqu'à émettre un jugement sur un passage, peut-être le plus deprauatus des commentaires $^{78}$, et sur lequel personne ne s'est vraiment penché. Lorsque César passe en revue les peuples présents à Alésia, il use, selon le commentateur, d'un procédé superbe, pulchra ratione, pour les énumérer, partant des plus puissants pour finir par les moins puissants ${ }^{79}$. Mais, en procédant au compte exact des peuples dénombrés par César, Glaréan s'aperçoit qu'à un certain moment ce nombre est assorti d'une approximation (circiter); il doit alors comptabiliser à son tour et plus précisément tous ces Gaulois réunis à Alésia. Ainsi, c'est lui, le commentateur qui fournit la liste complète et exacte des Gaulois regroupés dans la place forte, laissant à ceux qui sont attachés à la langue latine le soin de considérer comment César, pour établir une telle nomenclature des peuples gaulois, a employé les distributifs de façon appropriée et conforme à l'elocutio latine ${ }^{80}$.

Ces qualités d'eloquentia de César, le lecteur peut les identifier à nouveau dans deux des 73 rubriques que Glandorp consacre au commentaire du livre VII ${ }^{81}$. Alors que le danger est grand pour les Gaulois qui s'avancent vers Gergovie, Litaviccos, rassemble ses soldats et s'adresse à eux, en larmes : Quo proficismur, inquit, milites? Et le commentateur propose l'analyse suivante : «le discours est insidieux, son exorde est abrupt et dominé par l'interrogation » avant de préciser que César a su trouver les modes d'expression appropriés (selon les règles de la conuenientia) aux sentiments de l'orateur, douleur et indignation, compensant ainsi la transgression des règles du genre délibératif ${ }^{82}$. Glandorp analyse également le discours de Critognat ${ }^{83}$ :

\footnotetext{
${ }^{78}$ Par deprauatus il faut entendre «à corriger». C'est ce qui ressort de propos écrits par Guarino et cités par Silvia Rozzo (Il lessico filologico degli umanisti, Sussidi Eruditi 26, Roma, 1973, p. 223) : minus deprauatum habebis uolumen, nam in multis locis emendaui.

${ }^{79}$ II, p. 121. Imperant Haeduis atque eorum clientibus (B.G. VII, 75). Notandum itaque est Coesarem oppido pulchra ratione hanc descriptionem instituisse, nempe ut potentissimos quosque populos primum enumeraret, deinde minus potentes postponeret.

${ }^{80}$ II, p. 122. Obiter etiam hoc Latince linguce studiosis considerandum quam Latine et proprie Coesar distributiuis, ubi debuit, tota hac populorum nomenclatura utatur.

${ }^{81}$ I. Glandorp, In Librum septimum Commentariorum C. Iulii Caesaris de Bello gallico Annotationes. II, p. 146-49.

${ }^{82}$ II, p. 148. Quo proficismur (B.G. VII, 38). Oratio insidiosa est, habetque exordium abruptum, et ab interrogatione ductum, quale conuenit dolore et indignatione plenis. On sait que l'exorde, qui vait pour fonction de retenir l'attention des auditeurs, devait être prononcé dans un style simple et retenu. Mais Cicéron, qui préconise que cet exorde soit rarement véhément (De oratore II, 317) use précisément de cette véhémence, dans sa première Catilinaire, choisissant le mode de l'altercatio (reposant sur l'interrogatio) et conservant ainsi, aux dires de Quintilien (I. O. IX, 2), toute la fougue qui fut la sienne lors de la première séance au sénat, où il invectiva Catilina, alors présent.

${ }^{83}$ II, p. 149. (B.G. VII, 77). Oratio Critognati consultationem et confirmationem habet, ut decet eum, qui respondet et arguit, quemadmodum prcecipit Fabius lib.5. Confutat enim sententiam eorum qui eruptionem faciendam censebant, docetque breui affuturas popularium copias. Deinde censet in obsidione persistendum et tolerandum, ac humanis corporibus uescendum potius, quam deditionem faciendam, quae seruitutem allatura sit. Et est Deliberatiui generis oratio. En appelant «discours délibératif» ce que Müller dénommait «suasoire»,
} 
César, en choisissant le genre délibératif, organise bien les parties du discours délibératif, consultatio ${ }^{84}$, confirmatio, confutatio ${ }^{85}$, sachant adjoindre la breuitas là où il le faut. Le commentateur, quand bien même ses notes sont souvent succinctes, est attentif au style de César, remarquant une construction insolite mais non fautive, coutumière de César ${ }^{86}$, un archaïsme ${ }^{87}$, et n'hésitant pas à marcher sur les pas de G. Budé ou de Glaréan pour attester le choix heureux ou malheureux de tel ou tel mot. Toutefois si la langue de César n'est pas exclue du commentaire, celui-ci se veut plus explicatif des sous-entendus historiques, des termes spécifiques, voire techniques, ou encore des noms géographiques, de façon à ancrer les commentaires dans la contemporanéité du lecteur ${ }^{88}$.

De Joachim Camerarius, seules les annotations de quelques livres sont citées : ce maigre excerptum ${ }^{89}$ laisse apparaître cependant qu'il s'apparente à l'enarratio antique ; c'est une donnée historique, technique ou stylistique qui repose moins sur une paraphrase personnelle que sur des citations d'auteurs classiques, latins et grecs. Les remarques donnent lieu quelquefois à une actualisation, topographique notamment, telle celle qui précise que Vacalus (B.G. III, 10) dont l'orthographe varie d'une édition à l'autre désigne tout simplement l'endroit aujourd'hui appelé Walin. Enfin, elles prennent en compte les éditions déjà parues, telles celle de Beatus Rhenanus ou encore de Glaréan, ce qui confirme bien le critère chronologique retenu par Jungerman pour disposer ses sources.

La seconde famille regroupe essentiellement des paléographes et codicologues :

Glandorp ne fait qu'utiliser un synonyme puisque selon Quintilien (I.O. III, 8), il y a identité entre la pars deliberatiua et la suasoria qui impliquent pareillement le suadere et le dissuadere.

${ }^{84}$ Selon la Rhétorique à Herennius I, 2, il existe trois genres de causes, le démonstratif, le délibératif, le judiciaire : le genre délibératif consiste en une action délibérative, qui comporte persuasion et dissuasion. Deliberatiuum est in consultatione, quod habet in se suasionem et dissuasionem

${ }^{85}$ Selon la Rhétorique à Herennius I, 4, confirmatio est nostrorum argumentorum expositio cum adseueratione. La confirmation est l'exposé de nos arguments réalisé avec assurance. Confutatio est contrariorum locorum dissolutio. La réfutation consiste à anéantir tous les points antithétiques.

${ }^{86}$ II, p. 148. Cuius consensu (B.G. VII, 29). Consensu obsistere dixit in casu dandi, quemadmodum lib. 6. Casu locum relinquere. Solet autem Coesar ad hunc modum loqui, quemadmodum complura exempla docent, non in his tantum Commentariis, uerum etiam aliis Coesaris lucubrationibus et scriptis. Vide A. Gellium lib. 4 cap. 16.

87 II, p. 148. Huic rei praeuertendum (B.G. VII, 33). Huic rei praeuertendum dixit constructione ueteribus usurpata, pro quo mos dicere solemus: Mandatis rebus prcenerti uolo.

${ }^{88}$ Comme dans les remarques de Müller, les vocables géographiques sont proposés avec une orthographe variable et presque toujours fournis sous l'appellation contemporaine du commentateur. Exemple (p. 148): Ex oppido Cauillono (B.G. VII, 42). Id oppidum Ptolemaeus Cabullinum uocat, situm ad Ararim, hodie Galli sua lingua Chiallon uocant.

${ }^{89}$ II, p. 184-86. 
Giovanni Michaelo Bruto établit un relevé de uarice lectiones des Commentarii de César et fournit en exergue les codices auxquels il se réfère. Ce libellus ${ }^{90}$ qui regroupe diverses leçons d'un même passage des commentaires s'assortit de quelques éclaircissements sur les endroits obscurs. Il est précédé d'une indication sur les sigles $^{91}$ adoptés par Bruto pour désigner les codices dont il use ${ }^{92}$ et d'une invitation au lecteur de prêter une attention particulière aux leçons suivies d'un astérisque. L'observation de ces leçons fait apparaître que l'apparat de Bruto ne se limite pas à la citation de celles propres à chaque codex mais renvoie à certains autres éditeurs de César (Glaréan, Pierro Vettori ${ }^{93}$, Donato Gianotti ${ }^{94}$, Bartolomeo Ricci ${ }^{95}$ ). Bien plus, Bruto commente le choix qu'il opère, après comparaison des exemplaria dont il dispose, par le fait que les auteurs classiques ou encore la ratio et l'usus encouragent une leçon plutôt qu'une autre, que presque tous les manuscrits ont fait ce choix ${ }^{96}$, que le contexte césarien le justifie ${ }^{97} \ldots$

\footnotetext{
${ }^{90}$ I. M. Bruti Libellus Variarum Lectiones, in quo et loci plures obscuri in Casare explicantur. II, p. 187- 207. On sait que ce libellus n'est qu'une partie de l'œuvre consacrée par Bruto à César. Cf. supra, n. 48.

${ }^{91}$ C. Carrariensis codex, qui manuscriptus idem et antiquissimus est.

G. Gryphii, minimce formce, qui ex uetusto admodum exemplari est excusus Lugduni.

F. Impressus LX abhinc anno Florentice.

P. Parisiensis Roberti Stephani.

Vasc. Excusus Lutetice a Vascosano.

${ }^{92}$ On notera que, à l'exception du Parisiensis, les différents codices, sont précisés par des adjectifs qui les distinguent les uns des autres-manuscriptus, antiquissimus, excusus, impressus. Silvia Rozzo (op.cit., p. 69) signale que l'imprimé n'apparaît pas aux humanistes différent du manuscrit, notamment parce que les incunables reproduisent fondamentalement les caractéristiques du manuscrit (écriture, disposition sur la page, numérotation des feuillets...). La différence, pour eux, est essentiellement technique, l'imprimé répondant à un nouum scribendi genus.

${ }^{93}$ Ce Florentin (1499-1585) brilla de façon précoce par sa connaissance du grec, du latin et des mathématiques. Il séjourna à Rome où il fréquenta des archéologues. De retour dans sa cité, quand bien même il continuait de dénoncer les Médicis, il se vit confier par eux la chaire d'éloquence grecque et latine. Ce fut un philologue très productif dont on retiendra surtout les Variarum lectionum libri XXXVIII, composés à la façon des Nuits Attiques d'Aulu-Gelle, qui foisonnent de passages empruntés aux auteurs grecs et latins (d'après l'article de Weiss dans Bibliographie universelle ancienne et moderne, t. XLVIII, p. 330-32.)

${ }^{94}$ Connu davantage comme théoricien politique, exilé de la République florentine après le retour au pouvoir des Médicis, Donato Gianotti (1492-1573), installé à Venise, est l'auteur de deux traités relatifs à chacune de ces deux Républiques, Florentine et Vénitienne.

${ }^{95}$ Ce jésuite natif de Ravenne (1490-1569) fut précepteur à la cour de Ferrare. Il s'appliqua à l'étude de tous les genres de la littérature grecque et latine, depuis la comédie jusqu'à la rhétorique, en passant par la poésie.

${ }^{96}$ II, p. 195. Continebat (B.G. VII, 11) C. P. haud dubium quin contigebat sit legendum, quod ratio et usus docet et in omnibus fere habetur, et excus. et manuscript.*.

${ }^{97}$ II, p. 196. B.G. VII, 47.
} 
L'édition d'Aldo Manuzio junior ${ }^{98}$ ne propose que 28 gloses pour le livre VII : le souci de celui qui dirige l'imprimerie vaticane est de recenser les différences de lecture qu'il constate dans les deux Parisienses et le Gryphius principalement, mais aussi dans les livres de Giovanni Vincento Pinelli ${ }^{99}$, de Nicaise Ellebaudt ${ }^{100}$, dans l'édition d'Orsini ou encore dans le manuscrit qu'il cite comme antiquus li$b e r^{101}$ de la bibliothèque Vaticane. Il lui arrive de procéder à une correction, soulignant l'absence d'un mot (abest uerbum), d'une préposition notamment ${ }^{102}$. Il signale quelquefois, sans commentaires, des leçons différentes, ou les assortit d'une brève mention : non admodum probo, ou bien non mihi displicet, ou encore placet. Toutefois, le professeur de littérature et de rhétorique qu'il est s'en remet la plupart du temps aux auteurs latins eux-mêmes, César mais aussi Salluste, Virgile, Tite-Live, Végèce qui cautionnent les leçons qu'il propose. Et, au-delà de la critique textuelle, se laisse deviner une remémoration littéraire comme celle que suggère la glose consacrée à la relative quoe sit necesse accidere uictis ${ }^{103}:$ l'éditeur critique renvoie au discours que Salluste prête à César dans son De Coniuratione Catilince ${ }^{104}$, en rapportant longuement le propos du sénateur. La citation de la relative fait revivre la séance dans laquelle César interpellait ses concitoyens qui allaient devoir se prononcer sur le sort des conjurés et opposait aux arguments sensibles et pleins d'artifice de ceux qui avaient parlé avant lui et invoquaient le sort inévitable des vaincus) une argumentation plus philosophique et plus civique qui rappelait aux sénateurs qui

${ }^{98}$ A. Manuzio, Scholia in C. Iulii Caesari De Bello gallico. II, p. 212-13.

${ }^{99}$ G.-V. Pinelli (1535-1601) possédait une magnifique bibliothèque, toujours enrichie de manuscrits rares et des meilleures éditions. Cet érudit, féru d'hébreu, de grec et de latin, s'intéressait à toutes les sciences et se montrait disponible à tous ceux qui venaient le consulter.

${ }^{100}$ Nicaise Ellebaudt (15..-1577), médecin, philosophe mais aussi poète latin, est surtout connu dans la République des Lettres pour sa traduction de Némésius d'Émèse (De natura hominis) en latin, chez Plantin, à Anvers en 1565.

${ }^{101}$ D'après le tableau de datation des codices qu'établit Silvia Rozzo à partir des adjectifs qui les caractérisent (op. cit., p. 151), ce manuscrit ne remonte pas au-delà du $\mathrm{X}^{\mathrm{e}}$ siècle, ce que confirment nos éditions modernes.

${ }^{102}$ II, p. 195. In prcesentia de obsidibus cauere (B.G. VII, 2). Abest particula de in Parisiensi libro.

${ }^{103}$ César, De Bello gallico, VII, 14. Vercingétorix, après de nombreux revers, réunit son conseil et lui montre comment il doit priver de ravitaillement César et ses troupes partis pour Avaricum. Pour gagner les siens à son avis, il les invite à se représenter la captivité et l'esclavage de leurs femmes et de leurs enfants, leur propre extermination, soit « ce qui arrive inéluctablement aux vaincus ».

${ }^{104}$ Salluste, De Coniuratione Catilince, 51 : César, invité à se prononcer sur le sort des conjurés, dénonce l'artifice et l'éclat des discours prononcés avant le sien et reproduit, non sans ironie, le tableau misérable qu'ils ont représenté pour dire «ce qui arrive aux vaincus»: Plerique eorum, qui ante me sententias dixerunt, conposite atque magnifice casum rei publica miserati sunt. Quce belli sauitia esset, quae uictis adciderent, enumerauere : rapi uirgines, pueros; diuelli liberos a parentum conplexu; matres familiarum pati quce uictoribus conlubuissent ; fana atque domos spoliari ; ccedem, incendia fieri ; postremo armis, cadaueribus, cruore atque luctu omnia conpleri. 
l'écoutaient leur véritable devoir politique. C'est ce que le lecteur, bien au fait de l'ensemble du discours sallustéen, peut lui aussi se remémorer au travers de la rubrique aldine.

Des spicilegia de Zsámboki ${ }^{105}$, c'est le livre VII qui fournit le plus nombreux recensement. Toutefois les 30 rubriques qui s'y réfèrent sont très brèves. Partant d'une expression césarienne, Zsámboki cite beaucoup le Vaticanus Codex (V.C.), proposant la leçon qu'il contient, émettant quelquefois un jugement. Mais il s'en tient surtout à ce qu'il appelle son ancien manuscrit (meus uetus codex ${ }^{106}$ ) qui lève les inquiétudes qu'il peut avoir en observant d'autres codices $^{107}$ ou lui permet de corriger des absurdités ${ }^{108}$.

De l'autre collectionneur de manuscrits qu'est Fulvio Orsini ${ }^{109}$, Jungerman a reproduit les remarques plus nombreuses que celles de Zsámboki. Les notes du livre VII sont au nombre de 47 et sont essentiellement codicologiques ${ }^{110}$ : Orsini, lui aussi, a entre les mains le Vaticanus codex et se limite au constat des différences qu'il révèle par rapport aux ueteres codices ou encore aux leçons de Faerno ${ }^{111}$, qu'il s'agisse de manques, d'ajouts, de ponctuation, d'orthographe des noms. Et lorsqu'il émet son propre avis, il est rarement tranché (ut fortasse legendum sit-quod non uidetur reiciendum / quod uidetur legendum / rectius/ adundate).

Enfin c'est dans un ensemble de 86 rubriques $^{112}$, qu'un autre philologue paléographe manifeste sa préoccupation de vérifier les différentes leçons jusqu'ici transmises. L'espagnol Chacon, devant la grande variété des codices de l'œuvre césarienne (Vaticanus, Florentinus, Parisienses, Gryphius...), traduit sa volonté d'établir au mieux le texte et de justifier les choix de son liber. Ainsi multiplie-t-il les legendum est/ legendum existimo, ou encore dénonce-t-il les lectures corrompues, les ajouts de mots (uox abundat /delenda est), accordant une grande importance à la lecture conjointe ou nom des mots (coniuncte legendum / uerbum superioribus iungi potest), soit à la ponctuation. La plupart du temps, pour justifier ses choix, il recourt à des exemples d'auteurs de la période classique (notamment Cicéron), d'écrivains spécialisés (Vitruve), à ceux des historiens les plus glorieux (Salluste, Tite-Live), sans oublier les références précieuses que constituent tous ceux qui se sont intéressés, en passant ou systématiquement, à la géographie (Pline l'Ancien, Strabon, Orose, Ptolémée et, bien plus tard, Marlianus). Car l'enarratio n'est pas étrangère à ses gloses : et si cette paraphrase vient souvent justifier tel ou

\footnotetext{
${ }^{105}$ In Comm. I. Caesaris Lectiones et Spicilegia qucedam I. Sambuci. II, pp. 229-30.

${ }^{106}$ D'après le tableau de S. Rozzo (op. cit. p. 151), ce manuscrit serait du XI'.

${ }^{107}$ II, p. 250. Atque omnia prius experiantur (B.G. VII, 78). Hic locus mihi scrupulum obiecerat, post me liberauit uet. c. lectio senior : Atque omnia prius expediantur quam ad Critognati sententiam descendant ; illo tamen tempore, utendum potius consilio, si res cogat etc.

${ }^{108}$ Ibid., Nostri emissis pilis gladiis rem gerunt (B.G. VII, 88). Videtur in loco angusto absurdum, uet. C. omissis pilis etc.

${ }^{109}$ Vrsini Notce ad librum septimum De Bello gallico. II, p. 243-44.

${ }^{110}$ Trois seulement font référence à des sources antiques, respectivement Salluste, Tite-Live et les Fasti Capitolini.

${ }^{111}$ Gabriele Faerno (1510-1560) était un philologue rigoureux, spécialisé dans la critique et la révision des éditions modernes des classiques latins.

${ }^{112}$ Ciacconii Notce in librum septimum de Bello gallico. II, p. 187-91.
} 
tel choix de lecture, parmi les codices observés, elle vaut quelquefois pour ellemême notamment lorsqu'elle éclaire le contexte historique et institutionnel (à propos de la uirtus de Pompée, par exemple ${ }^{113}$ ) ou les particularités géographiques et ethnographiques afférentes aux noms gaulois. Ainsi lorsque César évoque les Rutheni prouinciales $^{114}$, Chacon indique en passant qu'il faut ne pas faire de pause dans la lecture, et consacre tout le reste de sa glose à tenter de résoudre la difficile localisation de ce peuple gaulois (est-ce en Narbonnaise ? en Aquitaine ?), s'appuyant bien sûr sur César lui-même, Pline et les géographes anciens. Alors qu'il vient d'évoquer l'orthographe d'Alésia (Alexia? Alesia? Halosia ? ${ }^{115}$ ), différente selon les codices, il consacre la glose qui suit à la description de l'oppidum avant de proposer une lecture, qu'il justifie en citant Hirtius et César lui-même ${ }^{116}$. Les Notce de Chacon ne se réduisent pas à un commentaire critique des manuscrits ou éditions anciennes : elles constituent une sorte d'adéquation du commentaire antique aux questions que soulève la variété des lectures jusqu'ici opérées.

Si pour les éditeurs précédents, il s'agissait surtout d'établir le texte et de corriger des leçons antérieures en justifiant ou non cette correction, pour Chacon il s'agit de prendre appui sur toutes sortes de sources, manuscrites ou imprimées, et d'établir un texte qui fournit les données géographiques, historiques, techniques que le récepteur est en droit d'attendre, ce qui implique une exactitude de l'oratio latine, garantie si possible par les tenants de la latinitas et par les meilleurs spécialistes de la géographie et de l'histoire, quand bien même ceux-ci se trompent. Ce qu'atteste la rubrique qu'il consacre à la ville des Boïens que certains désignent sous le nom de Gergouia et que lui-même nomme Gergobina ${ }^{117}$.

C'est dans la même perspective que se situe Hotman qui fournit un très grand nombre de scolies pour ce livre VII de la Guerre des Gaules ${ }^{118}$; nombreuses sont

\footnotetext{
${ }^{113}$ II, p. 187. Virtute Cn. Pompei ( B.G. VII, 6). Is enim creatus consul post Clodii caedem legibus ferendis iudiciisque faciendis Remp. Constituerat. «Deorum uirtute » dicere solebant. Plaut. Aulular. Chacon cite deux vers de Plaute, se réfère à Salluste et à César pour justifier l'expression coutumière deorum uirtute.

${ }^{114}$ II, p. 188. B. G. VII, 7.

115 II, p. 190. B.G. VII, 7. La première orthographe qui est celle des codices Vrsinus et Vaticanus diffère de celle qu'ont choisie Dion Cassius et Plutarque. Quant à la troisième, on la lit chez Orose.

${ }^{116}$ Ibid., Sub muro quae pars collis ad Orientem spectabat (B.G. VII, 69). Qua collibus cingebatur Alesia, ipsa loci natura satis tuta erat, sed qua patebat III mil. passuum planicies, ipsce Gallorum copice consederant et fossam maceriamque sex in altitudinem pedum produxerant. Quare suspicor legendum hic esse: quce pars collium in circuitu uacabat. Sic enim loquitur Hirtius (B.G. VIII, 41) ab ea parte, quae fere pedum trecentorum interuallo a fluminis circuitu uacabat. Sic quoque Coesar (B.G. VII, 70) in ea planitie, quam intermissam collibus tria milia passuum in longitudinem patere supra demonstrauimus.

${ }^{117}$ II, p. 188. Gergouiam Boiorum opidum (B.G. VII , 9). Aut aliud est opidum Gergouia in Aruernis quo Vercingetorix expulsus est, supra in principio huius libri, et quod obsidet Caesar infra : aut hoc loco Gergouia corrupte legitur et, ne quid dissimulem, a Strabone Aruernorum Гepyovı dicitur, nisi codex fallit. Sed hoc loco meus codex habet Gergobinam Boiorum et Paris. codex, uide Marlianus ; alias Gergouiam semper.

${ }^{118}$ F. Hotmanus, In Librum VII De Bello gallico Notce. II, p. 370-95.
} 
celles qui concernent l'établissement du texte, en référence aux éditions antérieures et plus particulièrement à celle de Manuzio, d'Orsini, de R. Estienne, de Faerno. Lorsqu'il expose les leçons de ses prédécesseurs, qu'il les retienne ou non, Hotman ne justifie son choix que de façon très laconique et, s'il lui arrive de proposer une leçon toute personnelle, il ne se résout pas à l'adopter s'il n'a pas la garantie d'un $\operatorname{codex}^{119}$. En revanche, il n'hésite pas à réfuter les lectures des autres éditeurs lorsqu'il privilégie la latinitas de l'expression et par là-même son sens exact, et il oublie même qu'il établit un texte de sorte qu'il poursuit sa glose sur le mode de l'enarra$t_{i o^{120}}$. En effet, soucieux d'expliquer le texte de César, citant à l'occasion comme modèles Asconius ou Servius, il reformule volontiers, et selon sa propre interprétation, la pensée de César, à la façon des commentateurs antiques ${ }^{121}$. Nous lisons alors des notes plus développées qui guident une lecture «vraie » grâce au respect de telle règle grammaticale ${ }^{122}$, de données historiques ${ }^{123}$, géographiques (celles-ci pouvant résulter d'une interprétation toute personnelle ${ }^{124}$ ou étayée de références empruntées à des spécialistes anciens ou modernes) ou encore de quelque tactique militaire ${ }^{125}$. Enfin, attentif à la lettre du texte, Hotman assortit certaines remarques linguistiques d'un jugement stylistique. Il observe le choix des mots et, s'il dénonce quelques usurpationes, il souligne bien plus souvent la qualité de l'écriture césarienne: c'est l'emploi propre du mot ciuitas, par exemple, que nulle

\footnotetext{
${ }^{119}$ II, p. 371. Eo tempore pacati (B.G. VII, 6). Suspectus mihi locus. Fortasse non satis pacati, sed sine codicis auctoritate, nihil mutabo. In Romano est Quieti.

${ }^{120}$ II, p. 389. Silentio excepit (B.G. VII, 28). Vrsinus Silentio sic ex fuga recepit, neque aliter sensum putat elici potest idoneum. Ego animaduerti aliud esse, Recipere ex fuga, aliud Excipere ex fuga. Illud est, cum qui fugiebant, ad locum destinatum peruenerunt: hoc uero, cum in ipsa uia et fuga intercipiuntur, ut supr. libr. $6 \mathrm{ca}$. 7 : A quibus receptos ex fuga Tenctheros atque Usipetes supra docuimus. Et mox: Primos Eburonum fines adeunt; multos ex fuga dispersos excipiunt. Vercingetorix ergo de illorum fuga certior factus celeriter familiares suos principes ciuitatum obuiam illis misit, qui silentio ipsos ad amicos suos deducerent.

${ }^{121}$ II, p. 389-90. Ad Caesarem disceptatorem (B.G. VII, 37). Vrsino et Farn. Placet ex antiquo lib. Disceptatorem. Ego idem uideo in lib. Steph. Neque tamen acquiesco. Nam utrocumque modo addendum esset, de suo, hoc modo Quam Romani de suo ad Aeduos. Itaque uocem Romani tollendam arbitror, ut sit sensus : Cum de iure et legibus Aeduorum ageretur, quid ita iudicium eius rei potius penes Casarem, quam penes ipsos Aeduos fuisset? Significat Cosarem ea de re iudicasse, qua non fuerat ipsius iudicii: ac propterea ei acceptum magistratum ferri non debere.

${ }^{122}$ II, p. 370. Hinc alii (B.G. VII, 3). Steph. Hanc alii. Ego Hunc, ut ad clamorem referatur.

${ }^{123}$ II, p. 371. Virtute Cn. Pompeii (B.G. VII, 6). Turbata cade Clodii ciuitate, cum seditiones inter candidatos consulatus Hipsceum, Scipionem et Milonem essent, qui armis ac ui contendebant, ad comprimendas eas Cn. Pompeius a senatu consul III factus est absens et solus : quod nulli alii unquam contigit. Epit. 107. Donnée historique empruntée à la Periocha CVII de Tite-Live.

${ }^{124}$ II, p. 370. Tota prouincia (B.G. VII, 1). Gallia Cisalpina.

${ }^{125}$ II, p. 372. Aggerem cuniculis subtrahebant (B.G. VII, 22). Opidani fossas subterraneas sub moenibus aperiebant usque ad aggerem Romanorum. Hinc terram subtrahebant ut turris in aggerem promota subuerterentur. Une note de même facture fournit la fiche technique des duce fossce dont César dit, au chapitre LVII, qu'il les fait creuser.
} 
part dans la Guerre des Gaules César n'utilise à la place de urbs ou de oppidum ${ }^{126}$; c'est la justesse d'une description, telle celle d'Avaricum, qui permet d'attester l'authenticité du lieu qu'en français nous appelons Bourges, tant le site réel convient de façon très appropriée - aptissime conuenit - à la description de César ${ }^{127}$; c'est la sécheresse aléatoire d'une autre expression comme id spatium itineris augebat: l'auteur a sans doute ajouté devant le verbe quelque chose pour représenter la distance, un altero tanto (doublement), ou un uehementer (considérablement) ou encore quelque chose de ce genre car, dit Hotman, «je ne pense pas que César ait écrit dans un style aussi sec sur une réalité évidente ${ }^{128}$. Le regard qu'Hotman porte sur les mots témoigne d'une excellente connaissance du texte césarien et les analyses qu'il propose de ce point de vue se réfèrent essentiellement à la consuetudo de César, sans doute parce que son commentaire procède d'une lecture assidue et enthousiaste des Commentarii $^{129}$. Et lorsqu'il considère qu'il y a quelque obscurité, c'est-à-dire, lorsqu'il ne «comprend pas » (locum a me non intellegi, ingenue fateor), il n'impute pas la faute à César - qui privilégie quelquefois, dit-il, la mens plutôt que les uerbamais à lui-même ou au mauvais établissement du texte : il procède alors à des réécritures explicatives ou «redresse » le texte transmis. On sent que même si son objectif premier est de retrouver le texte «vrai », de justifier ses propositions textuelles par la règle, grammaticale ou rhétorique (notamment pour résoudre des ellipses), il pénètre la pensée de César et va même jusqu'à lui prêter de l'ironie dans l'emploi d'un uirtute au lieu d'un ignauia ${ }^{130}$. Hotman, pour qui les indications en matière d'onomastique, de géographie, d'histoire, d'institutions sont utiles au lecteur, cherche tout autant à retrouver la mens de César au travers de ses uerba. Et lorsqu'il souligne, comme le fait Juste-Lipse ${ }^{131}$, certains emplois qui pourraient passer pour

\footnotetext{
${ }^{126}$ II, p. 370. Expellit ex ciuitate (B.G. VII, 4). Opinor non ex urbe sed Auernorum finibus. Nam Ciuitatem in his libris nusquam pro urbe opidoue positum animaduerti. A la page suivante, pour développer l'expression ciuitatem Biturigum, il rappelle que César, dans aucun de ses sept livres, n'emploie ciuitas à la place d'urbs ou d'oppidum.

${ }^{127}$ Hotman souligne ici deux qualités conjointes de l'art oratoire : à savoir la conuenientia et le sermo aptus.

${ }^{128}$ II, p. 390. Spatium itineris augebat (B.G. VII, 46). Non puto Ccesarem adeo ieiune de re perspicua scripsisse.

${ }^{129}$ Dans la Préface de son César, édité en 1574 chez V. Bartholomée - préface que ne reproduit pas Jungerman - Hotman déclare que, non seulement les Commentaires de César ne sont jamais tombés de ses mains, mais encore qu'ils ont détendu son esprit, comme s'il était sous un portique, ou même faisait une très agréable promenade. Et c'est à une contemplation de l'œuvre césarienne, à l'égale de celle que mérite une tabula, qu'Hotman invite son dédicataire.

${ }^{130}$ II, p. 372. Virtutem despicere (B.G. VII, 20). Ironice Virtutem appellat pro Ignauia. Une ironie que Quintilien reconnaît à César, rapportant dans son Institution oratoire (VI, 3, 90) des propos tenus par César, en sa qualité de témoin d'une femme que son mari avait malmenée.

${ }^{131}$ Dans son Electorum liber secundus (ch. VII), Juste-Lipse relève quelques inquinamenta, ou expressions sordides, qui attestent, selon lui, que les Commentaires ne sont pas aussi purs et louables qu'ils paraissent, de sorte qu'il apporte, preuves à l'appui, des corrections au texte même de César.
} 
des impropriétés, il assortit toujours sa remarque d'un opinor ou d'un potius qui modalisent son interprétation : tout en levant ainsi l'ambiguïté de sens qu'il suppose, tout en éclairant le lecteur de César, il constitue un petit album d'elegantice. Ainsi donc, le commentaire d'Hotman prend en compte la dimension critique du texte qu'il établit, mais démontre également que l'exégèse héritée de l'antiquité demeure indispensable pour éclairer la lecture de César au XVI .

C'est dans la même intention de proposer un texte bien établi et bien commenté que Johannes Brandt produit ses Notes Politiques et ses Notes Critiques, mais il se distingue d'Hotman par le fait qu'il organise son commentaires en deux cahiers distincts dont l'un s'apparente à une explication du texte, nourrie de références anciennes et modernes, le plus souvent édifiante pour le lecteur, et dont l'autre relève de la critique textuelle.

Les Notes Politiques de Brandt ${ }^{132}$, qui occupent le premier des deux cahiers, attestent un souci d'explication exhaustive, visant à conférer le plus de clarté possible au sens des expressions et passages retenus. Le lecteur est ainsi bien informé de tout ce qui touche à l'histoire et aux mœurs des Romains, - au travers de celles des Gaulois bien souvent - et surtout à leurs conceptions politiques en matière de droit et d'art militaire. Le mos maiorum et la libertas sont bien sûr envisagés dans le contexte de la guerre des Gaules mais le commentateur prolonge une analyse circonstanciée par des explications qui font référence à d'autres situations de guerre, d'autres citations d'exploits et de comportements militaires, transmis par les auteurs latins. Lorsqu'il est question, pour les Gaulois, au début du livre VII, de défendre la gloire et la liberté qu'ils ont héritées de leurs ancêtres, Brandt glose le texte en proposant une évidence - il vaut mieux mourir au nom de la liberté que de vivre dans la servitude ennemie - qu'il étaie de nombreuses citations empruntées à Salluste, à Cicéron, à Publius Syrus ou encore à Denys d'Halicarnasse, signalant de surcroît que ces citations ont été commentées par des contemporains (tels Muret, Vettori).

La plupart des passages glosés relèvent du domaine historique et militaire et leur exploitation, sans être détachée du contexte gaulois, sert une perspective politique - voire une idéologie - que Brandt a clairement annoncée dans la dédicace qu'il adresse aux magistrats et au peuple d'Anvers. Les deux notions conjointes de Iustitia et de Res militaris, que le commentateur a mises en exergue dans cette dédicace et qu'il a définies du point de vue anversois, sont latentes dans la plupart de ses remarques.

La Iustitia et la Res militaris, dit-il dans sa dédicace, sont indissociables, les lois servant d'auxiliaires à la vertu guerrière. La première est bien connue des Anversois : elle fait leur force chez les nations soumises autant que chez eux. Et à l'occasion de troubles civils - comme c'est le cas présentement - la rigueur à rendre le droit tout autant qu'une faveur juste, une écoute généreuse et patiente, une disponibilité de jour et de nuit sont à conserver par ces dédicataires dont les oreilles doivent être sourdes aux pleurnicheries et aux rumeurs. La seconde a été bien illustrée par ces mêmes Anversois qui se sont montrés à la hauteur des Anciens lorsqu'il s'est agi de verser leur sang, avec une vertu singulière, qu'ils ont mise à l'épreuve, par exemple, à l'endroit de Lier (Lira) le jour même où cette ville était prise d'assaut par

${ }^{132}$ II, p. 428-89. 
les Bataves. C'est en vertu de leur renommée universelle que l'Archiduc Albert et son épouse Isabelle ont adhéré à leur assemblée et choisi leurs conseillers.

Brandt poursuit cette dédicace en justifiant son choix de commenter César : les exemples de ceux qui, autrefois, se sont dévoués pour la cité l'ont stimulé en ce sens car c'est en sondant l'Histoire qu'il a le mieux compris les malheurs actuels et appris à les surmonter. Or, en matière d'historiographie, César est le premier d'entre tous parce qu'il a été à la fois soldat et chef et que sa pratique, nécessaire quand on écrit l'histoire, s'est conjuguée avec sa science militaire. Et c'est bien ce qu'illustrent ses Commentarii qui, de surcroît, touchent de près «à nos affaires » ditil, c'est-à-dire aux affaires de ces Belga dont le général Romain a bien dit qu'ils étaient, de tous les Gaulois, avec les Nerviens, les plus courageux. Sans ce courage, ils auraient multiplié querelles et factions auxquelles pas même César avec ses légions n'aurait pu mettre un terme. A ce moment de la dédicace, Brandt ouvre une parenthèse qui prolonge les simultates et factiones en question : ut plerumque fit cum rebus fractis et labentibus extremum malorum dicordia accedit ${ }^{133}$. Il met fin à son propos en déclarant que s'il a été encouragé par ses amis à commenter César et bousculé par le comportement de ses ennemis, c'est en faveur de la cité plutôt que de ses illustres gouvernants qu'il s'est acquitté de cette tâche $a b a_{c t i s}{ }^{134}$. Voilà pourquoi il produit ses commentaires en deux parties : l'une qui concerne la République, l'autre ceux qui sont versés dans les études.

Ainsi les concepts et les pratiques antiques sont actualisés de sorte que les contemporains de Brandt peuvent comprendre, à travers le texte de César, que tous les enjeux auxquels se sont heurtés les Gaulois ne sont pas très différents des leurs; ils sont invités à inscrire les troubles qui affectent leur pays dans une sorte de continuité avec les temps antérieurs. Brandt, en suggérant ce rapport au présent de ses lecteurs, rejoint d'autres érudits - dont Juste-Lipse - qui, engagés en politique, ont eu le souci de faire régner la justice dans un temps où la guerre divisait cruellement leurs concitoyens.

Quelques exemples suffisent à illustrer le mode de commentaire «politique», original dans l'ouvrage de Jungerman, conduit par Brandt. Au chapitre IV du livre VII de la Guerre des Gaules, il est question du père de Vercingétorix tué par ses compatriotes, suspecté d'avoir recherché le titre de roi. Brandt commence par énumérer d'autres peuples Gaulois, cités dans d'autres livres du Commentaire, et qui ont eu un roi. Suit alors un développement de son fait (notandum est) sur la transmission du trône : l'hérédité, de ce point de vue, ne compte pas chez les Gaulois qui lui préfèrent une désignation par l'assemblée du peuple, chaque nation étant accoutumée à déférer le pouvoir au nom d'une suprême justice et d'une suprême sagesse, iustitia prudentiaque summa. Pour attester cette coutume, le commentateur cite Ambiorix qui considère son statut tel «qu'il n'a pas plus de pouvoir sur la multitude que la multitude n'en a sur lui », avant de citer Strabon qui évoque dans sa Géogra-

\footnotetext{
${ }^{133}$ II, p. 417. «comme il se produit la plupart du temps lorsqu'à un État brisé et chancelant arrive en sus le pire des maux, la discorde».

${ }^{134}$ Tout en se situant à un niveau de «greffier», Brandt souligne le caractère officiel et juridique de sa démarche.
} 
phie les institutions politiques gauloises en matière de désignation de magistrats ou de généraux ${ }^{135}$.

Lorsqu'il s'agit de commenter la uirtus de Pompée, loin de citer la Periocha de Tite-Live, comme Hotman ${ }^{136}$, Brandt procède à un développement sur la uirtus des anciens qui se considéraient comme redevables de leurs succès aux dieux et à la vertu, ne voulant pas donner l'impression de se les attribuer à eux-mêmes et d'être ingrats envers les dieux. De la même façon ils estimaient qu'ils n'avaient pas à cacher la vertu des hommes vaillants. Dans cette note, la référence auctoriale est d'abord Turnèbe, un moderne, dont l'enseignement précède l'évocation par César $(B G, \mathrm{~V}, 52)$ d'un affront «vengé par le bienfait des dieux immortels et du courage des soldats ${ }^{137} »$.

Telle autre note exalte la bravoure guerrière et patriotique que récompense la couronne murale et que confirment de nombreux exemples héroïques ${ }^{138}$. Telle autre encore définit le général en chef courageux et vaillant ${ }^{139} \ldots$

\footnotetext{
${ }^{135}$ II, p. 429. Et ob eam causam, quod regnum adpetebat, a ciuitate erat interfectus (B.G. VII, 4). Aruernum regium imperium detrectabant, non item alii plerique Galli, Sequani Catalamandelem Regem habuere, ut est in initio libri primi de bel. Gal. Suessiones primum Diuiaticum, post Caesaris aduentum Galbam libro secundo, Aquitani Pisonis cuiusdam Aquitani auum libro quarto, Senones Moritasgum libro quinto. Nitiobriges Ollouicorum libro septimo. Notandum autem non fuisse hereditaria eiusmodi regna (quando Casar plures priuatos nominat, quorum tamen pa-rentes regnum in sua ciuitate obtinuissent) sed a populo in consiliis cuiusque gentis propter iustitiam sapientiamque summam deferri solita, cum imperio non illo quidem infinito et effrenato, sed certis legibus circumscripto, ut nec plenum ius nisi fortasse in bello haberent. Itaque Ambiorix Eburonum Rex ita loquitur libro quinto Sua esse imperia, ut non minus haberet iuris in se multitudo, quam ipse in multitudinem, ut uere Strabo libro quarto (cité en grec, puis traduit en latin: Aristocratice erant plerceque, Gallorum Resp. unum autem magistratum olim quotannis deligebat; sicut ad bellum gerendum unus a populo imperator creabatur).

${ }^{136}$ Cf. supra note 123.

${ }^{137}$ II, p. 429. Cum ille iam uirtute Cn. Pompeii (B.G. VII, 8). Sicut Deûm uirtute dicebant ueteres cum res prosperas Diis et uirtuti acceptas referant, ne sibi eas attribuere uiderentur, et in Deos ingrati esse: sic nonnunquam etiam hominum uirtutem tacitam non prcetermittebant, ut docet Adr. Turneb. lib. 2 aduers. c. 29 et lib. 18 ca. 7. supra lib. 5 : quod beneficio Deorum immortalium et uirtute eorum (militum) expiato incommodo.

${ }^{138}$ César (B.G. VII, 27) pour encourager ses soldats propose une récompense à ceux qui auront franchi le mur les premiers. Cette proposition est tout d'abord glosée par le biais d'Aulu-Gelle et de sa définition de la couronne murale. Puis c'est Tite-Live qui est cité lorsqu'il évoque l'attribution de cette couronne par Scipion, après la prise de Carthage avant que soit fait un renvoi d'une part aux exemples qu'a recensés M.A. Muret et qui illustrent cette récompense, d'autre part au catalogue de couronnes qu'a établi Juste-Lipse.

${ }^{139}$ II, p. 461. Id tamen se celeriter maioribus commodis sanaturum (B.G. VII, 29). Fortis et strenuus imperator si quid in bello detrimenti acceptum est, id breui tempore posse sarciri cogitat. Itaque non statim hastam abiicit, neque unius proelii euentu pauescit, sed operam dat, ut acceptum incommodum uirtute reparetur, uires iuxta et procul sitas circumspicit, nouos delectus, noua qucerit auxilia, in summa omnia experitur, more anguium qui obtrito capite postremum cauda minantur ut de Mithriditate scribit L. Florus lib. 1 c. 5.
} 
Sans conteste, les commentaires de Brandt sont guidés par l'idée maîtresse de sa dédicace, à savoir que la conjonction de la justice et de l'art de la guerre doit être la force de tous les Belgae. Les circonstances dans lesquelles Brandt compose ses Notes politiques sont celles d'une guerre civile et religieuse pour laquelle l'archiduc Albert et son épouse Isabelle cherchent à obtenir une trêve. C'est donc dans une perspective, sinon d'unification, du moins du respect du droit de la guerre qu'il commente César, si célèbre pour sa clémence. Tel est l'engagement politique de Brandt (tel était aussi celui de son ami Juste Lipse dont il déplore la mort toute récente $^{140}$ ) qui donne sens au commentaire du texte de César et il semble bien que Jungerman lui-même, qui laisse au juriste anversois la primeur d'utiliser les notes de Chacon dans la partie «critique», partage la générosité de son contemporain, quand bien même il ne partage pas ses choix religieux.

Brandt, apôtre de justice, ne rompt cependant pas avec ses prédécesseurs et, comme tout érudit de l'époque, ajoute à une série de sententice politica ${ }^{141}$, empruntées à César, et qui suivent ses Notce Politicae, des Notce critica ${ }^{142}$. Ces dernières, moins copieuses que les notes politiques, procèdent d'une méthode comparative et dérivative identique à celle dont usaient au siècle précédent les philologues soucieux d'établir au mieux le texte césarien. Elles se distinguent cependant par la multiplicité des citations et des références qui les accompagnent et par la coexistence de commentateurs lointains et proches : depuis Donat, commentateur de Térence, jusqu'à Juste-Lipse en passant par Manuzio, Beroaldo, Budé, Chacon, Hotman, Vascosan... Brandt semble englober ici toute une tradition en s'appuyant néanmoins sur des textes imprimés plus que sur les manuscrits eux-mêmes. De toute évidence, l'établissement du texte qui selon lui intéresse essentiellement la Res litteraria, sans être négligeable, est second par rapport à une perspective politique qui intéresse la Res publica. Les circonstances invitent à faire des Commentaires un objet de réflexion politique et Brandt se les approprie en ce sens, au risque de les faire passer pour prétextes.

L'interprétation libre, mais sincère, de Brandt n'est pas de son seul fait, quand bien même il la revendique de façon franche et délibérée ; en effet, de façon

\footnotetext{
${ }^{140}$ Juste Lipse (1547-1606) est mort à Louvain en mars 1606. Ce catholique d'origine, converti au protestantisme, est revenu au catholicisme en 1591. La notice qui lui est consacrée dans le Dictionnaire de Pierre Bayle (vol. 3 : 122-126) qui semble presque entièrement fondée sur ce revirement, est particulièrement dépréciative, allant jusqu'à souligner qu'il fut même accusé de bigoterie. Ce retour au catholicisme s'est accompagné d'un engagement aux côtés de Philippe II d'Espagne, puis de l'archiduc Albert qui l'a désigné comme son conseiller en raison de son expérience de la justice, suggère Brandt sans sa dédicace.

${ }^{141}$ II, p. 489-491. Les expressions retenues sont la plupart du temps modifiées ou augmentées pour obtenir un véritable statut de sententice. Par exemple, lorsque Vercingétorix (B.G. VII, 4) se montre d'une excessive sévérité avec ceux qui ont commis une faute grave "pour qu'ils servent d'exemple à tous les autres et que la grandeur du châtiment inspire à ceux-là de la terreur », Brandt ajoute un Puniendi delinquentes, il faut punir ceux qui ont manqué à leurs obligations ut sint reliquis documento et magnitudine poence perterreant alios.

${ }^{142}$ II, p. 491-535. Ces notes sont suivies d'un index qui regroupe, selon un ordre alphabétique, les expressions commentées, avec un renvoi à l'œuvre et au chapitre dont elles sont extraites.
} 
plus discrète, la plupart des autres commentateurs se laissent guider par un leitmotiv ou une fantaisie qui laissent à penser que leurs notes «récupèrent » quelque peu les textes de César. Et nous pouvons quelquefois nous demander pourquoi le sens à donner à certains passages varie tant de l'un à l'autre (cf. uirtus de Pompée), si le texte n'est pas au service d'une visée subjective, telle celle du «nationaliste » Glaréan, ou d'une passion, telle celle du traqueur de manuscrits qu'est Bruto. Assurément, il ne faut pas oublier que tous ces commentateurs ont été réunis dans ce suntagma par le choix - libre ou imposé - du compilateur et que, sortis de leur ensemble, associés de façon arbitraire (car chronologique), certains «livrets » ont été affectés d'une nouvelle fonction (celle de participer d'une publication collective décidée par un tiers) et par là-même d'une portée sans doute différente de celle d'une publication isolée. Quoi qu'il en soit, leur assemblage permet au lecteur moderne de regarder César, tel qu'il a été reçu depuis la première moitié du XVI ${ }^{\mathrm{e}}$ siècle jusqu'à l'aube du XVII ${ }^{\mathrm{e}}$, sous des facettes multiples, au gré de chaque commentateur.

Aussi bien Müllers que Brandt, reproduits au début et à la fin de la «file»des commentateurs, soulignent respectivement dans leur Préface et leur Dédicace la place qu'occupe César dans l'historiographie. La définition du genre du commentarius par le premier, mais aussi par plusieurs qui lui succèdent dans l'ouvrage ${ }^{143}$, la préséance que lui accorde le second, sous caution de Cicéron, en le mettant à la tête de la familia historica, sont la preuve que l'auteur des Commentarii occupe une place de choix dans ce domaine. Il apparait aussi comme un garant de la latinitas en ce qu'elle a de plus sobre et de plus exact, quand bien même sa qualité exceptionnelle d'elegantia, souvent citée en exergue des notes et annotations, et parfois mise à l'épreuve dans les commentaires paraphrastiques, est étrangère aux paléographes. Chez ceux-là, il semble en effet que les textes de César répondent plutôt à leur zèle infatigable à collectionner ou à collationner des manuscrits. N'oublions pas, en outre, que les Commentarii fournissent de précieuses indications qui servent, sinon de cautions, du moins de références aux géographes ou aux ethnographes. Enfin, grand observateur des hommes et de leurs techniques guerrières, César est aussi un habile tacticien militaire, un imperator dont le carnet de bord, nous l'avons vu, peut fournir

\footnotetext{
${ }^{143}$ Plusieurs de nos commentateurs définissent le commentaire en général, d'après les anciens, et retiennent qu'il fournit de la matière. En effet, alors que le genre relève de l'éphéméride pour certains écrivains de l'antiquité (Plutarque, Servius), pour d'autres il s'apparente plutôt à l'hupomnema, composé memorice gratia, c'est-à-dire de façon que soit gardée en mémoire la matière rapportée. Les deux perspectives envisagent essentiellement la materia, dont les humanistes savent bien que, dans le genre du commentaire, tel qu'il a été défini par N. Perotti, elle est présentée de façon sommaire. Müller (II, p. 3) cite en effet la définition de Perotti : Commentarius est liber in quo rerum modo capita posita sunt, ita ut uideamur in illis ad exequendam historiam, aliis subiecisse materiam. Toutefois, la plupart des sources réunies par Jungerman considèrent que si César livre bien pour mémoire une matière à la postérité, il y met un soin tel que ses Commentarii diffèrent du genre traditionnel. Et même s'il ne se conforme pas à la norme d'une écriture de l'histoire pleine et exacte, choisissant seulement de livrer sommairement les faits, il n'en ressort pas moins, aux yeux de Glaréan et de Glandorp entre autres, qu'il se situe entre deux historiens reconnus, l'un pour sa breuitas (Salluste), l'autre pour sa copia (Tite-Live).
} 
à Brandt des exemples de vertu militaire utiles à ses contemporains pour qu'ils ne se départissent pas, dans leurs luttes intestines, de leur pratique notoire de la Justice.

En nous mettant en contact avec tant de commentateurs et éditeurs des Commentarii, Jungerman nous a permis de voir également la variété de leur pratiques intellectuelles et souvent - notamment chez les héritiers des peuples qu'avait soumis César - à côté d'une certaine volonté de se montrer rigoureux dans l'établissement du texte, une appropriation bien subjective des récits et des descriptions que ce texte fournit. Le choix du compilateur a été guidé par les occasions qui se sont présentées à lui de se procurer toutes ces notes, mais ses convictions religieuses et les circonstances politiques dans lesquelles il a produit son ouvrage n'ont sans doute pas été étrangères à sa réalisation. Ce petit-fils de Joachim Camerarius, correcteur à l'imprimerie Wechel, nourri des idées de la Réforme, ne semble cependant pas avoir fait prévaloir un parti pris. En effet, au-delà de son entreprise nouvelle de commenter une traduction grecque - tout aussi nouvelle - des Commentarii, il n'a pas refusé la demande qui lui était faite de rassembler des commentateurs; il les a associés quelle que fût leur obédience, protestante ou catholique, sans hésiter à placer ses «modestes» annotations de la traduction grecque à la suite des notes d'un Anversois catholique, notes copieusement reproduites et augmentées d'une dédicace à des Princes catholiques ainsi que d'un éloge à l'endroit du jésuite Schott. Si Jungerman a d'abord songé à servir la Respublica litteraria, il n'a pas craint pour autant, à sa façon, de servir la Res publica: tel le pilote qui, transportant César à Brindes, désespérait de lui faire faire la traversée par peur d'une mer démontée, notre éditeur a vraisemblablement perçu cette exhortation : «Courage, car tu transportes César ! ${ }^{144} »$.

Monique BOUQUET CELAM - Université de Rennes II

\footnotetext{
${ }^{144}$ Cette comparaison est suggérée par Jungerman lui-même qui commence ainsi sa Dédicace à Paul Petau (I, p. 2): Gubernator nauis, Vir amplis. in qua CAESAR, milites suos, qui Brundisii substiterant, accersere uolens ipse, uehebatur, transfretaturum se desperans, ne montes aquarum, neu scua uentorum flabra, tempestuosumque mare exhorrescat iubetur ab eximio suo epibate, scilicet quod CAESAREM uehat. Jungerman reproduit esnuite le cri

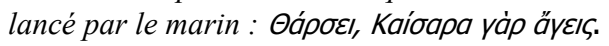

